\title{
A quantitative deconstruction of the morphology of high-latitude ionospheric convection
}

\author{
A. Grocott, ${ }^{1}$ S. E. Milan, ${ }^{1}$ S. M. Imber, ${ }^{1}$ M. Lester, ${ }^{1}$ and T. K. Yeoman ${ }^{1}$ \\ Received 30 January 2012; revised 3 March 2012; accepted 27 March 2012; published 17 May 2012.
}

[1] We present an analysis of ionospheric convection data derived from velocity measurements made by the Super Dual Auroral Radar Network (SuperDARN). Our analysis uses an established technique for combining the network data to produce maps of large-scale convection by fitting a spherical harmonic expansion of the ionospheric electric potential to the radar measurements. We discuss how the basis functions of the spherical harmonic expansion describe different characteristic elements of the ionospheric convection pattern and show how their associated coefficients can be used to quantify the morphology of the convection, much like the total transpolar voltage is used to quantify its strength, in relation to upstream interplanetary magnetic field conditions and associated magnetospheric activity. We find that $\sim 2 / 3$ of the voltage associated with the typical convection pattern is described by a simple twin vortex basis function. The magnitude of the twin vortex is strongly dependent on IMF $B_{Z}$ and the degree of its (typically westward) rotation is weakly dependent on IMF $B_{Y}$. The remaining $\sim^{1 / 3}$ of the total voltage is associated with deviations from the basic twin vortex pattern, introduced by the addition of other basis functions, such as IMF $B_{Y}$ associated dusk-dawn asymmetries, nightside convection features associated with tail activity, and "reverse" high-latitude convection cells associated with intervals of IMF $B_{Z}>0$.

Citation: Grocott, A., S. E. Milan, S. M. Imber, M. Lester, and T. K. Yeoman (2012), A quantitative deconstruction of the morphology of high-latitude ionospheric convection, J. Geophys. Res., 117, A05317, doi:10.1029/2012JA017580.

\section{Introduction}

[2] Magnetospheric dynamics are largely driven by the solar wind via coupling between the terrestrial and interplanetary magnetic fields. The large-scale effects of this coupling are readily observable in the ionosphere as auroral emissions, magnetic perturbations, and the pattern of ionospheric convection. The ability to observe this convection pattern has long been recognized as essential to our understanding of the solar wind-magnetosphere interaction and various techniques to provide large-scale observations have been developed. One of the most successful means of deriving the near-instantaneous large-scale convection pattern is afforded us by the Super Dual Auroral Radar Network (SuperDARN) [Greenwald et al., 1995; Chisham et al., 2007]. SuperDARN provides near-continuous coverage of a significant portion of the polar, auroral and subauroral ionospheres [e.g., Chisham et al., 2007] and is therefore ideally suited to studying the temporal variability of ionospheric convection and its relationship to season, geomagnetic activity and solar cycle.

\footnotetext{
${ }^{1}$ Department of Physics and Astronomy, University of Leicester, Leicester, UK

Corresponding author: A. Grocott, Department of Physics and Astronomy, University of Leicester, Leicester LE1 7RH, UK. (a.grocott@ion.le.ac.uk)

Copyright 2012 by the American Geophysical Union. 0148-0227/12/2012JA017580
}

[3] A number of previous studies have used SuperDARN to investigate the large-scale characteristics of ionospheric convection. Ruohoniemi and Greenwald [1996] performed the first such study using data from a single HF radar, in which they qualitatively described the statistical average patterns of convection for different levels of geomagnetic activity and different strengths and orientations of the interplanetary magnetic field (IMF). Ruohoniemi and Greenwald [2005] subsequently extended this study to incorporate data from additional radars and to include the effects of season and solar cycle. More recently, Pettigrew et al. [2010] investigated dipole tilt and interhemispheric dependencies, while Grocott et al. [2010] considered the effects of magnetospheric substorms on the evolution of the convection pattern. In addition to these somewhat qualitative investigations, a number of studies have used more quantitative global measures of the convection derived from the SuperDARN data. Shepherd et al. [2003], for example, considered how the strength of the convection varied with the solar wind electric field, using a measure of total transpolar voltage obtained from the SuperDARN observations, while Grocott et al. [2009] investigated the relationship between solar wind magnetospheric Mach number and driving efficiency by using the transpolar voltage as a proxy for the dayside reconnection rate.

[4] Recently, studies have increasingly focused on asymmetries in the coupled magnetosphere-ionosphere system [e.g., Sandholt and Farrugia, 2007; Laundal et al., 2010; Shi 
et al., 2010; Grocott et al., 2010; Lukianova and Kozlovsky, 2011]. Such asymmetries are intrinsically linked to the mechanisms that drive the dynamics of the system and in order to fully understand these mechanisms the asymmetries they introduce must be fully understood. In terms of ionospheric convection, a simple quantification of asymmetry is not as obvious as, say, using the transpolar voltage to quantify its strength. Studies looking at flow directions in the cusp, for example, can simply use point vector measurements to ascertain direction and hence asymmetry [e.g., Milan et al., 2000], whereas a quantification of large-scale asymmetry is less straightforward to conceive.

[5] Attempts at such quantitative analysis have been made using various data sets and techniques. For example, Sun et al. [1998] performed a mathematical separation of directly driven and unloading components of the ionospheric equivalent currents during substorms using the method of natural orthogonal components (MNOC). This technique, described by Kendall et al. [1976], separates the structure of (in their case) the geomagnetic field into different wavelength components which can then be directly compared to other parameters. Similar analyses using MNOC have been conducted to investigate the morphology of the aurora in association with solar wind parameters and IMF components [Baker et al., 2003] and to decompose the daily geomagnetic variation into the solar quiet daily variation and disturbance daily variation [Xu and Kamide, 2004]. A preliminary investigation of ionospheric convection using this technique was performed by Baker and Greenwald [2003] who found the first three eigenmodes of the convection to correspond to dusk-dominant, dawn-dominant, and nightside convection, respectively.

[6] An alternative method of describing the large-scale ionospheric convection pattern involves a spherical harmonic expansion of the ionospheric electric potential (see section 3 for a detailed description of this technique). Unlike $\mathrm{MNOC}$, in which 'natural' orthonormal basis functions are determined from the data set under analysis, spherical harmonics uses basis functions which are predefined. This has the advantage that the basis functions are independent of the data set and, as a consequence, can be readily reproduced for different data sets aiding comparative analyses. The main disadvantage, on the other hand, is that the physical significance of the different basis functions is not necessarily well understood. In the present paper, we investigate how the spherical harmonic coefficients derived from an established SuperDARN convection mapping analysis technique describe the morphology of the ionospheric convection pattern and show how they can be used to quantify both the shape and strength of the convection in relation to upstream interplanetary magnetic field conditions and magnetospheric dynamics. These results will enable further detailed investigations of the nature of ionospheric convection in response to different driving conditions and help elucidate the nature of the associated magnetospheric forcing.

\section{Instrumentation}

\subsection{Super Dual Auroral Radar Network}

[7] The Super Dual Auroral Radar Network (SuperDARN) is an international array of HF coherent radars in the Northern and Southern Hemispheres [Greenwald et al., 1995; Chisham et al., 2007]. In their normal operating mode, each radar scans through 16 beams of azimuthal separation $3.24^{\circ}$, with a dwell time of 3 or $7 \mathrm{~s}$ on each beam, thus completing a full $\sim 52^{\circ}$ scan in $\sim 1$ or $\sim 2 \mathrm{~min}$. For consistency, we use $2 \mathrm{~min}$ averages of the $1 \mathrm{~min}$ data to produce a continuous, regular time series of data at $2 \mathrm{~min}$ resolution. Line-of-sight Doppler velocity measurements are made on each beam at $45 \mathrm{~km}$ range resolution out to ranges in excess of $3000 \mathrm{~km}$. The shape and extent of the fields of view of the Northern Hemisphere SuperDARN radars that were in operation between the years 2000 and 2006 are illustrated in Figure 1. This figure shows the radar fields of view in a magnetic latitude-magnetic local time (MLT) coordinate system at 00:00 UT, with magnetic local noon to the top and dusk to the left. More complete coverage of radars in the Northern Hemisphere informed our decision to focus on these data, although as coverage in the Southern Hemisphere continues to improve, future interhemispheric studies should become feasible.

\subsection{Advanced Composition Explorer}

[8] Interplanetary magnetic field (IMF) data have been provided by the Magnetic Field Experiment (MFE) [Smith et al., 1998] on the Advanced Composition Explorer (ACE) spacecraft [Stone et al., 1998], which provides measurements of the IMF at $16 \mathrm{~s}$ resolution. Two minute averages of these data have been used to provide a data set contemporaneous with the SuperDARN observations. These data have then been time lagged to the dayside ionosphere using the method of Khan and Cowley [1999], which involves calculating the propagation delay of field changes from ACE to the dayside ionosphere and includes the propagation time in the solar wind upstream of the bow shock, the frozen in-transit time across the subsolar magnetosheath, and the Alfvènic propagation time along open field lines from the subsolar magnetopause to the ionosphere. The mean and standard deviation time lags derived for our data interval are $63 \mathrm{~min}$ and $10 \mathrm{~min}$, respectively.

\section{Data Processing}

[9] The analysis technique used in this study is based on the SuperDARN convection mapping algorithm described by Ruohoniemi and Baker [1998]. This analysis involves the expansion of the ionospheric electric potential, $\Phi$, in terms of spherical harmonic functions, $Y_{l m}$, given by

$$
\Phi(\theta, \phi)=\sum_{l=0}^{L} \sum_{m=-l}^{l} A_{l m} Y_{l m}(\theta, \phi)=\sum_{l=0}^{L} \sum_{m=-l}^{l} A_{l m} P_{l}^{m}(\cos \theta) \mathrm{e}^{i m \phi},
$$

where $A_{l m}$ are the complex coefficients of the expansion, $l$ and $m$ are the order and degree of the expansion, respectively, $P_{l}^{m}$ are the associated Legendre polynomials, and $\theta$ and $\phi$ are the magnetic colatitude and local time, respectively. The order up to which the expansion is performed $(L)$ is generally chosen according to the requirements of a given study. To resolve small-scale features in the convection, for example, relatively high expansion orders of 6 or 8 may be used. For the purposes of the present study, however, we use only the first 15 coefficients (up to 4th order) which, as will be shown below, are more than sufficient to describe the 


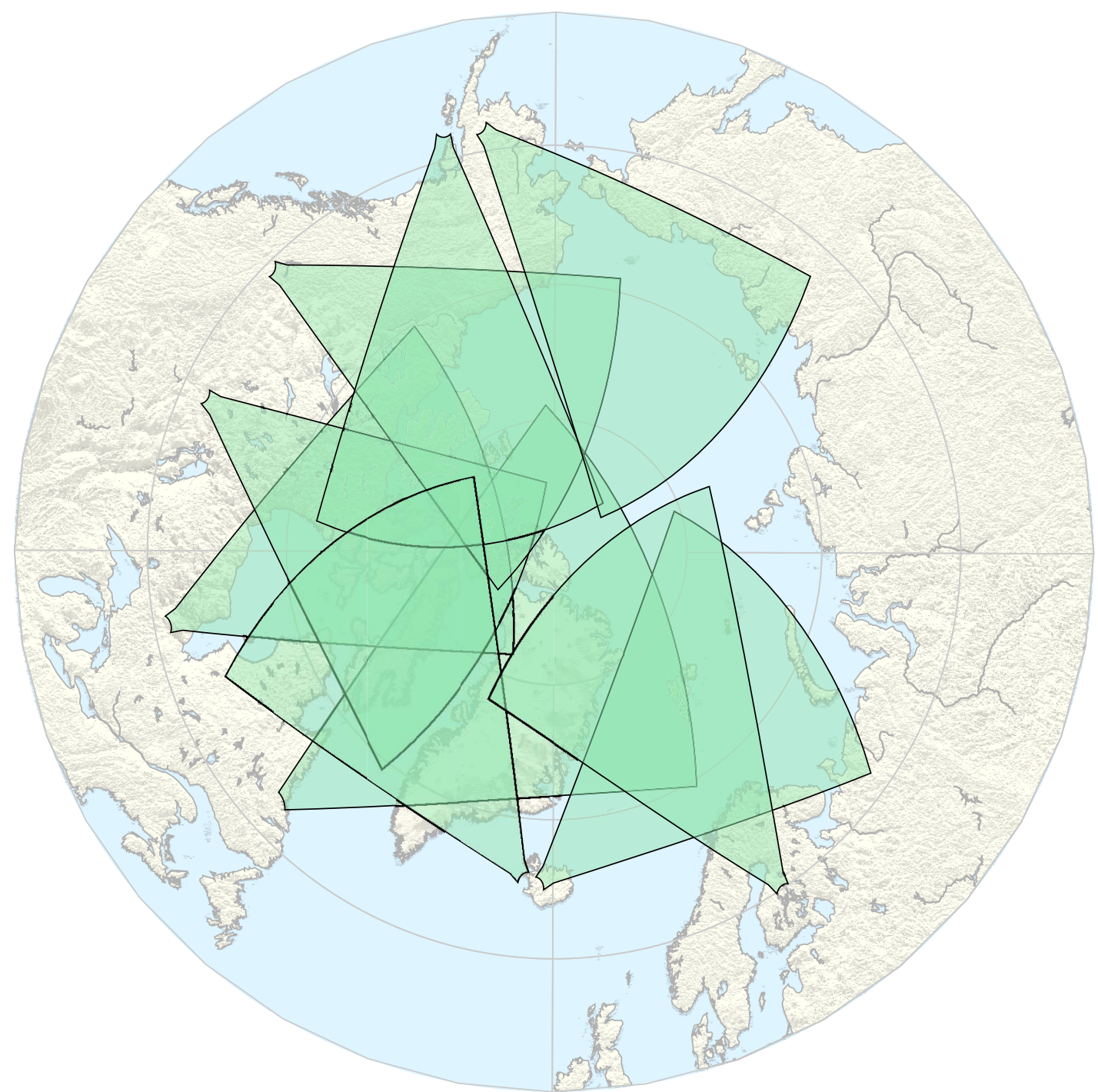

Figure 1. A map of the Northern Hemisphere polar cap and auroral zones showing the fields of view at 00:00 UT of the SuperDARN radars that were in operation between the years 2000 and 2006. Noon is to the top of the map, with dusk to the left, and the map extends down to $50^{\circ}$ magnetic latitude. Anticlockwise from noon the names of the radars are King Salmon, Kodiak, Prince George, Saskatoon, Kapuskasing, Goose Bay, Stokkseyri, Pykkvibaer, and Hankasalmi.

large-scale features under consideration. In practice, because the electric potential is assumed to be zero at low latitudes, an effective colatitude, $\theta^{\prime}$, is used in equation (1) which maps the latitude range of the electric potential over the full range of $\theta$ :

$$
\theta^{\prime}=\frac{\pi}{\left(\pi / 2-\Lambda_{0}\right)} \theta
$$

In this case, $\Lambda_{0}$ is the latitude at which the radar observations suggest the convection electric field goes to zero [Shepherd and Ruohoniemi, 2000].

[10] For reference, Figure 2 provides a set of polar projected electric potential patterns, on an arbitrary latitude scale, that are illustrative of the shapes of the first four order basis functions associated with each of the coefficients. The influence of each basis function on any given solution of $\Phi(\theta, \phi)$ is determined by the amplitude of the corresponding coefficient, $\left|A_{l m}\right|$, and its argument, $\operatorname{Arg}\left(A_{l m}\right)$ (subsequently referred to as $\left.\phi_{l m}\right)$ which governs its phase, or rotation in magnetic local time. In Figure $2\left|A_{l m}\right|$ has been artificially set to enhance the potentials in each case and $\phi_{l m}$ set to zero. In reality $\left|A_{l m}\right|$ decreases with increasing order and degree and $\phi_{l m}$ varies between $\pm 12 / m \mathrm{~h}$, as shown in section 4.1 below. This figure also serves to illustrate how the level of structure that can be resolved in latitude and local time increases with each step in order and degree. For $L=4$ fitting, as used here, this corresponds to a spatial resolution of the order of $1000 \mathrm{~km}$ (the exact value depending on latitude, and on $\Lambda_{0}$ ).

[11] To determine the values of $A_{l m}$ used in this study, we perform a fit to the 7 years of Northern Hemisphere SuperDARN data described in section 2.1. For each $2 \mathrm{~min}$ 

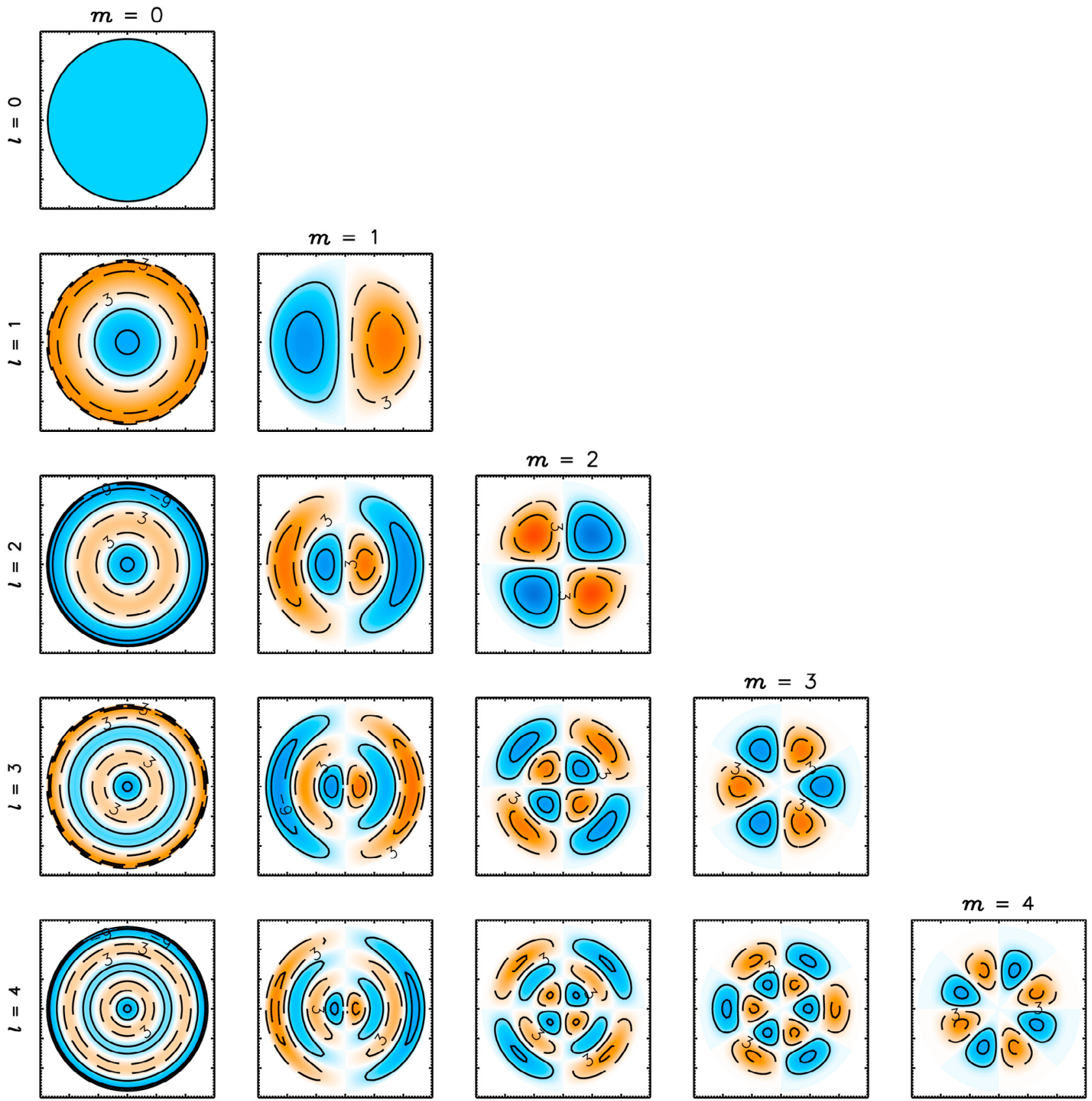

Figure 2. A set of polar projected electric potential patterns, on an arbitrary latitude scale, that illustrate the first four order basis functions associated with each of the coefficients $A_{l m}$. Increasing order, $l$, is from top to bottom and increasing degree, $m$, is from left to right. The magnitude of the potentials, $\left|A_{l m}\right|$, has been artificially set to enhance the features in each case and the rotation of the basis functions, Arg $\left(A_{l m}\right)$, is set to zero.

interval of radar data the line-of-sight velocity measurements are first filtered onto an equal area polar grid, where each grid cell is approximately a square of side length equivalent to $1^{\circ}$ of latitude, to produce a set of $N$ velocity values and their uncertainties, $W_{i}$ and $\sigma_{i}$. To fit the electric potentials described by the coefficients to the radar data it is necessary to represent them as an equivalent set of velocity vectors using the relation $\mathbf{V}=(\mathbf{E} \times \mathbf{B}) / B^{2}$ where $\mathbf{B}$ is the ionospheric magnetic field and $\mathbf{E}$ the electric field, given by
$\mathbf{E}=-\nabla \Phi$. The best fit convection pattern is then that which results in a global minimization of $\chi^{2}$

$$
\chi^{2}=\sum_{i=1}^{N} \frac{1}{\sigma_{i}^{2}}\left[\mathbf{V}[i] \cdot \hat{\mathbf{k}}[i]-W_{i}\right]^{2},
$$

where $\mathbf{V}[i]$ is the fitted velocity vector at the grid cell position associated with $i$ and $\hat{\mathbf{k}}$ is the line-of-sight direction of the radar beam associated with $W_{i}$. To ensure that a 

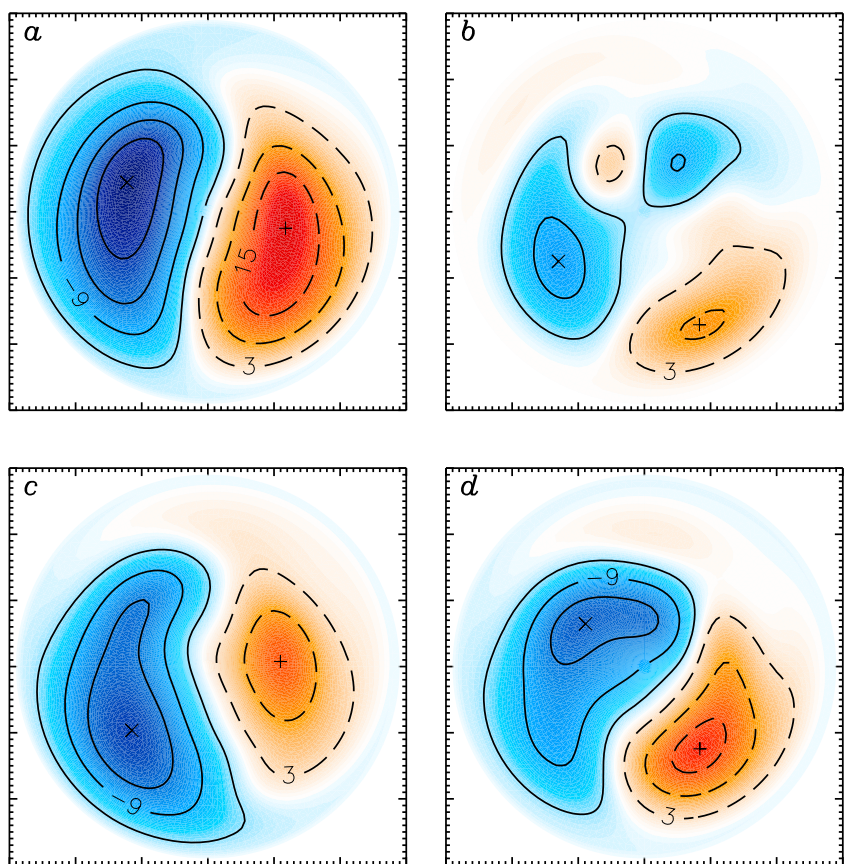

Figure 3. Four example ionospheric convection patterns associated with different dominant orientations of the IMF: (a) "twin vortex" convection, typically associated with a southward IMF $\left(B_{Z}-\right)$, (b) "reverse-cell" convection, typically associated with a northward IMF $\left(B_{Z}+\right)$, (c and d) asymmetric twin cell convection, typically associated with an east-west directed IMF (in the Northern Hemisphere, $B_{Y}-$ and $B_{Y^{+}}$, respectively).

sufficient spatial distribution of radar measurements exists to constrain the fitting process, additional velocity vectors taken from the statistical convection models of Ruohoniemi and Greenwald [1996] are added to the set of $N$ gridded velocity measurements prior to performing the fit. These model patterns are classified according to IMF strength and orientation and the appropriate model is chosen in each case via inspection of concurrent interplanetary data (see section 2.2). By this method, data from all radars in the Northern Hemisphere, often covering much of the polar cap and auroral zones, are combined to produce a single largescale convection pattern every $2 \mathrm{~min}$, that is completely described by a set of spherical harmonic coefficients and a boundary latitude, $\Lambda_{0}$. It should be noted that the sampling of model vectors in the SuperDARN fitting algorithm is designed to minimize their impact on the solution while still stabilizing the solution over areas of no radar observations. This minimization is effected by sampling the minimum number of points needed to constrain each term of the spherical harmonic expansion and by deweighting the sampled model vectors relative to direct measurements as described by Shepherd and Ruohoniemi [2000]. Nevertheless, to further minimize the dependence of the fit on the model vectors we restrict our subsequent analysis to intervals in which the number of gridded velocity vectors, $N$, is at least 300. Any remaining significance of the use of model vectors is discussed further in section 4.2 below.

[12] A set of four example convection patterns derived using this procedure are presented in Figure 3. These patterns illustrate the different geophysical scenarios observed under four different orientations of the IMF. In each case, the convection patterns are presented in the same reference frame as Figure 1, with noon to the top and dusk to the left. Their latitudinal extent (i.e., their size) is arbitrary in this case. Figure $3 \mathrm{a}$ illustrates a fairly typical twin cell convection pattern, with antisunward flow over the polar cap, often observed during intervals of southward IMF $\left(B_{Z}-\right)$ [e.g., Ruohoniemi and Greenwald, 2005]. Figure 3b illustrates the so-called 'reverse cell' convection pattern where, in addition to the 2 cells exhibiting antisunward flow over the polar cap, there are two smaller cells on the dayside circulating in opposite senses, notably producing sunward flow in the noon sector. This is a convection scenario that has been observed under strongly northward IMF $\left(B_{Z^{+}}\right)$[e.g., Imber et al., 2006, 2007]. Figures $3 c$ and $3 d$ show examples of twin vortex convection that exhibit a strong dawn-dusk asymmetry, understood to be introduced by the influence of IMF $B_{Y}$ (in the Northern Hemisphere, $B_{Y^{-}}$and $B_{Y}+$, respectively) [e.g., Ruohoniemi and Greenwald, 2005]. These patterns are discussed further in the next section.

[13] Lastly, we define two additional quantities used in our subsequent discussion in sections 4 and 5 . First is the maximum ionospheric potential difference, $\Phi_{\text {diff }}=\Phi_{\max }-\Phi_{\min }$, which provides a good proxy for the total transpolar voltage. As described above, $\Phi_{\text {diff }}$ is often used as a measure of the strength of the convection. We also define the residual of the positive and negative potential, $\Phi_{\text {res }}=\Phi_{\max }+\Phi_{\min }$, which provides a measure of the asymmetry in the potential, typically between the dawn and dusk twin vortex convection cells. It should be noted, however, that owing to the derivation of both these quantities being based solely on the potential distribution within the global solution of $\Phi$, their physical significance cannot be rigidly defined. The implications of this are discussed further in section 5.2.

\section{Observations}

\subsection{Coefficient Distributions}

[14] Figure 4 presents the occurrence distributions of the spherical harmonic coefficients, $A_{l m}$, for every $2 \mathrm{~min}$ Northern Hemisphere SuperDARN convection map for the years 2000-2006 in which at least 300 gridded radar vectors were available for the fit. These limits yield almost 500,000 maps which form the basis of our statistics. For $m=0$, where no rotational dependence exists (see Figure 2), the occurrence distribution is plotted as a histogram. For $m>0$ the distributions are shown versus the amplitude $\left(\left|A_{l m}\right|\right)$ and rotation angle $\left(\phi_{l m}\right)$ of the corresponding basis function shown in Figure 2. In this case the occurrence is shown on a normalized scale from blue to red according to the color bar on the right. It should be noted that the axis ranges are different in each plot in order to provide reasonably consistent distribution densities in each case. In addition, $\phi_{l m}$ (in hours) is plotted either symmetrically about $0 \mathrm{~h}$, or about $12 / \mathrm{m} \mathrm{h}$, depending on the typical polarity of the corresponding basis function. The black symbols superposed on each plot indicate the values of $A_{l m}$ for the four convection patterns shown in Figure 3: plus, $B_{Z}-$ (Figure 3a); diamond, $B_{Z}+$ (Figure 3b); triangle, $B_{Y^{-}}$(Figure 3c); and square, $B_{Y^{+}}$(Figure $3 \mathrm{~d}$ ). Figure 5 presents the occurrence distribution histograms of 

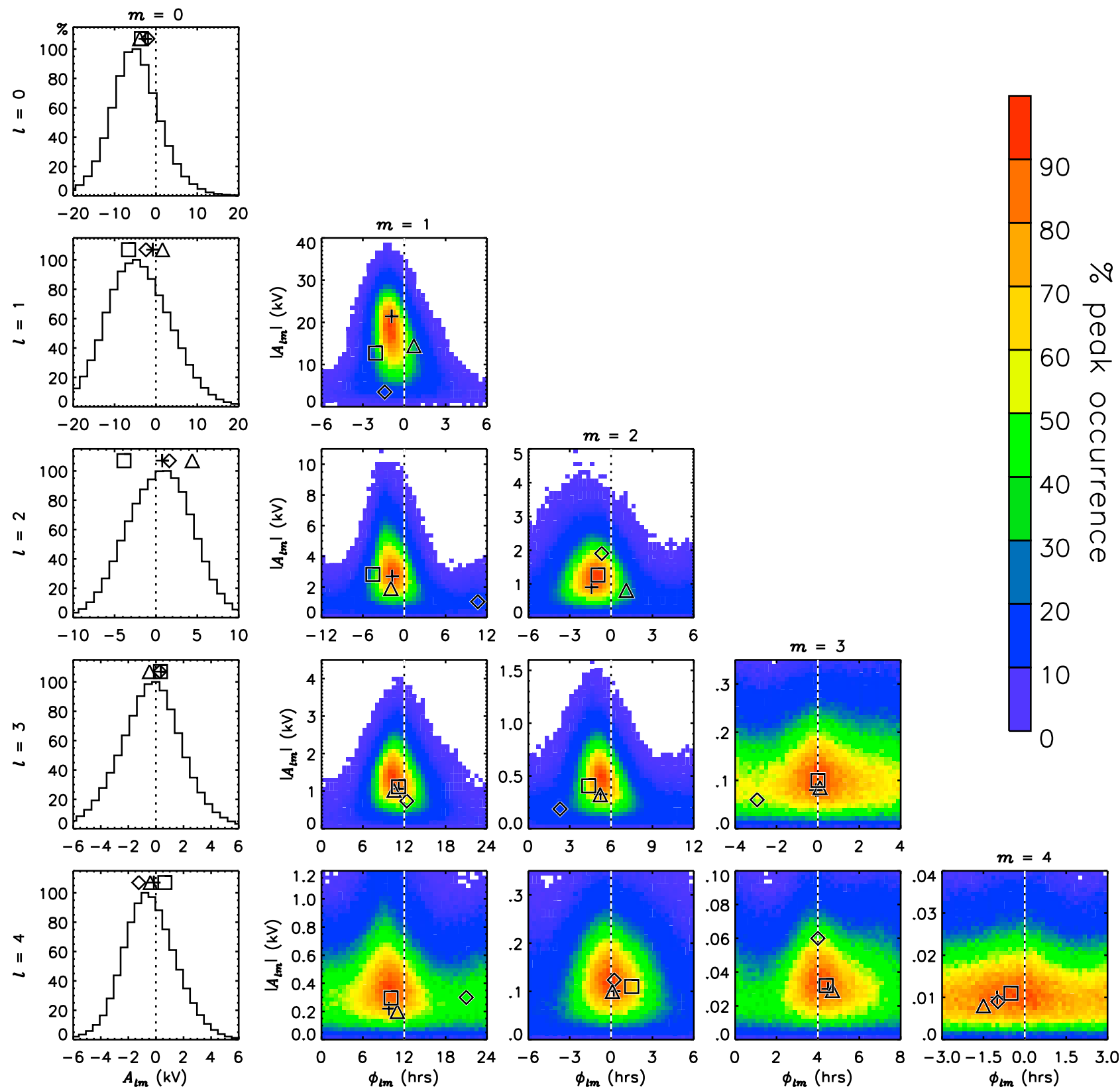

Figure 4. Occurrence distributions of the spherical harmonic coefficients, $A_{l m}$, discussed in the text. Where no rotational dependence exists, the distributions are shown as histograms, otherwise they are shown versus the amplitude $\left(\left|A_{l m}\right|\right)$ and rotation angle $\left(\phi_{l m}\right)$ of the corresponding basis function shown in Figure 2. Here the occurrence is shown on a normalized scale from blue to red according to the color bar on the right. Indicated by the black symbols are the values of the coefficients for the four representative convection patterns shown in Figure 3: plus, $B_{Z^{-}}$(Figure 3a); diamond, $B_{Z}+$ (Figure 3b); triangle, $B_{Y}-$ (Figure 3c); and square, $B_{Y}+$ (Figure 3d).

$\Phi_{\text {diff }}$ and $\Phi_{\text {res }}$, in a similar format to the $A_{l, 0}$ histograms in Figure 4.

[15] The data presented in Figure 4 reveal a number of characteristics of $A_{l m}$ and illustrate how they relate to the different geophysical scenarios presented in Figure 3. By far the strongest influence on the shape of the patterns can be attributed to the $A_{1,1}$ coefficients, evidenced by a mean amplitude of $15.6 \mathrm{kV}$, more than three times greater than that of any other coefficient. Figure 2 reveals that $A_{1,1}$ describes a basic twin vortex convection pattern which, according to, e.g., Ruohoniemi and Greenwald [2005], forms the basis of the convection pattern under almost all geophysical conditions. This is supported by a high correlation of $r=0.92$ between $A_{1,1}$ and $\Phi_{\text {diff, }}$, and the fact that the peak-to-peak voltage associated with $A_{1,1}$ (i.e., $31.2 \mathrm{kV}$, twice the amplitude) is $\sim^{2 / 3}$ of the mean value of $\Phi_{\text {diff }}(46.5 \mathrm{kV})$. The remaining $\sim 1 / 3$ of $\Phi_{\text {diff }}$ must, therefore, be associated with deviations from the basic twin vortex pattern introduced by the addition of other basis functions. It is these additional 

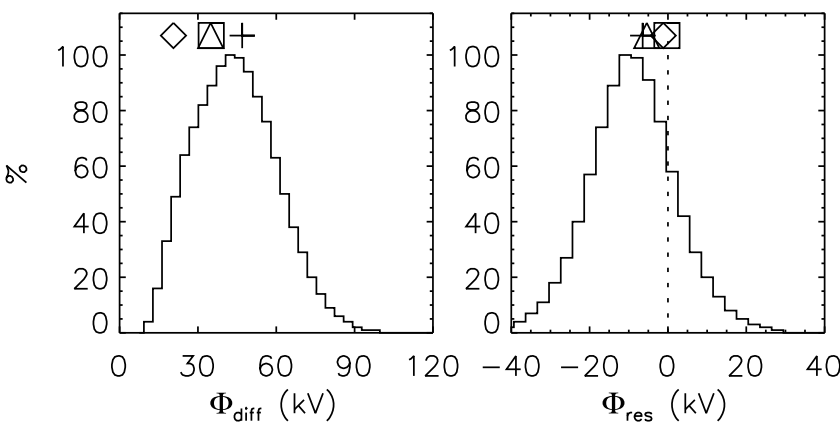

Figure 5. Occurrence distribution histograms of $\Phi_{\text {diff }}$ and $\Phi_{r e s}$, in the same format as the $A_{l, 0}$ coefficients in Figure 4.

contributions, and the physics that controls them, that we are particularly interested in.

[16] Also of significance is the range of $\phi_{\operatorname{lm}}$ over which $A_{1,1}$ is distributed. Although the tails of the distribution extend to $\pm 12 \mathrm{~h}$, in over $99 \%$ of cases $\phi_{1,1}$ falls within the $\pm 6 \mathrm{~h}$ range illustrated in Figure 4 . This is consistent with the polarity of the dawn and dusk cells almost always being positive and negative, respectively. It is interesting to note, however, a modest negative shift of the $\phi_{l m}$ distribution by $1-2 \mathrm{~h}$, corresponding to a clockwise rotation, indicating the general trend of the convection in the polar cap to be oriented slightly away from the noon-midnight meridian toward earlier MLTs. The separation of the square and triangle symbols in $\phi_{l m}$ is also consistent with an IMF $B_{Y}$ influence on the rotation; this is addressed further in section 4.2 below. Lastly, the separation of the plus and diamond symbols (corresponding to the $B_{Z^{-}}$and $B_{Z^{+}}$cases, respectively) indicates that the amplitude of $A_{1,1}$ is directly related to the strength of the twin vortex convection, with the pattern shown in Figure $3 \mathrm{a}$ (plus, $B_{Z}-$ ) exhibiting much stronger convection than that shown in Figure $3 \mathrm{~b}$ (diamond, $B_{Z}+$ ).

[17] The other key difference between the convection patterns shown in Figures $3 \mathrm{a}$ and $3 \mathrm{~b}$, in addition to the difference in convection strength, is in the number of convection cells. The introduction of two additional high-latitude cells, typically during intervals of northward IMF, is clearly associated with the 2nd order basis functions shown in Figure 2 , in particular, those described by $A_{2,1}$ and $A_{2,2}$. By examining the location of the diamond $\left(B_{Z}+\right)$ symbol in Figure 4 it would appear that an increase in $A_{2,2}$ amplitude is responsible for introducing the two additional cells, with the $A_{2,1}$ amplitude actually below average. However, the rotation of the $A_{2,1}$ basis function by $12 \mathrm{~h}$ seems to be critical in determining that the low-latitude cells retain the polarity of the original twin vortex convection, while the high-latitude cells exhibit the opposite polarity of the 'reversed' convection associated with lobe reconnection [Imber et al., 2007]. It is interesting to note that the amplitudes of both coefficients are also nonzero for the simple twin vortex $/ B_{Z}-$ case (plus symbol). At low amplitudes it would appear that the effect of these coefficients is to simply push the twin vortex pattern to slightly higher latitudes $\left(A_{2,1}\right)$ and to offset it from the pole toward the nightside $\left(A_{2,2}\right)$. The latter effect has been shown observationally in numerous studies of the highlatitude convection [e.g., Heppner and Maynard, 1987].
[18] The zero degree coefficients $\left(A_{0,0}, A_{1,0}\right.$ etc. $)$ describe additional modifications to the convection pattern that typically introduce dusk-dawn asymmetries. Consider the first coefficient, $A_{0,0}$, for example. The tendency for this coefficient to be negative is likely responsible for the typical dominance of the dusk convection cell [e.g., Ruohoniemi and Greenwald, 2005]. Indeed, an associated negative bias is also evident in $\Phi_{\text {res }}$ (shown in Figure 5) with a strong correlation existing between $A_{0,0}$ and $\Phi_{r e s}(r=0.88)$. The tight grouping of the four symbols (representing the different patterns) in this case suggests, however, that the $A_{0,0}$ coefficient has little further significance in controlling variations in the shape of the convection pattern. The second coefficient, $A_{1,0}$, has a similar distribution to $A_{0,0}$. It can be easily deduced from Figure 2 that the addition of these two basis functions acts to reinforce the negative potential at high latitudes while simultaneously weakening it at lower latitudes, essentially maintaining a physically realistic reduction in the potential, with decreasing latitude, to zero at the equatorward boundary. This is consistent with a relatively high correlation between $A_{0,0}$ and $A_{1,0}$ of $r \approx 0.84$. However, the four symbols are less closely grouped in this case; the triangle and square, representing the two IMF $B_{Y}$ dominated patterns from Figure 3, are notably separated (as they are in the $A_{2,0}$ case) indicating that these coefficients play a more significant role in describing IMF $B_{Y}$ related asymmetries in the potential patterns. This is discussed further in section 4.2.

[19] As one looks to the higher-order coefficients, and in particular the higher degrees, the amplitudes of $A_{l m}$ become small (typically less than $1 \mathrm{kV}$ ) and their distributions become broader. This indicates a reduced significance of these coefficients in governing the nature of the convection patterns, as might be expected. In terms of the example patterns from Figure 3, the diamond (representing the $B_{Z}+/ 4$ cell pattern in Figure $3 b$ ) tends to be displaced from the other symbols in the distribution, with a modest separation in $\phi_{l m}$ of the square and triangle $\left(B_{Y}+/ B_{Y}-\right.$ cases in Figures 3c and 3d) still apparent. However, in general the example cases lie relatively close to the modal values, indicating that on average the variability evident in these coefficients is not strongly contributing to the nature of the convection patterns for the simple cases illustrated in Figure 3.

\subsection{IMF Dependence}

[20] The example convection patterns presented in Figure 3 and discussed above, were chosen based on a qualitative appreciation of the IMF control of the shape of the ionospheric convection pattern. In this section, we consider this relationship quantitatively by investigating the dependence of a subset of $A_{l m}$ on the strength and orientation of the IMF. Figure 6 presents distributions of the first six coefficients (Figures 6a-6i) binned according to concurrent values of IMF $B_{Y}$ (left to right, -20 to $+20 \mathrm{nT}$ ) and $B_{Z}$ (top to bottom, +20 to $-20 \mathrm{nT}$ ). This presentation also illustrates the IMF clock angle dependence, where the clock angle is given by $\theta=\arctan \left(B_{Y}, B_{Z}\right)$ and is defined for $-180^{\circ}<\theta \leq 180^{\circ}$. Figure $6 \mathrm{j}$ shows the IMF occurrence distribution of the data. Bins containing fewer than 5 data points have been excluded from the presentation of the coefficient data shown in Figures 6a-6i to reduce the noise at more extreme IMF conditions. In all other bins the mean value of the 


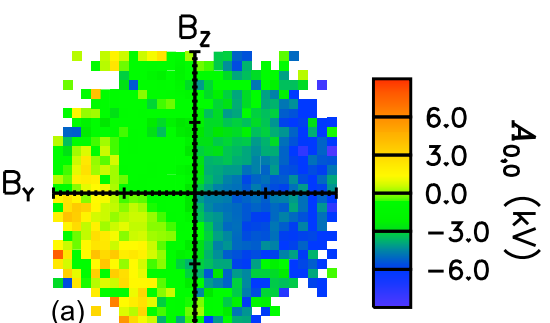

(a) $=2$
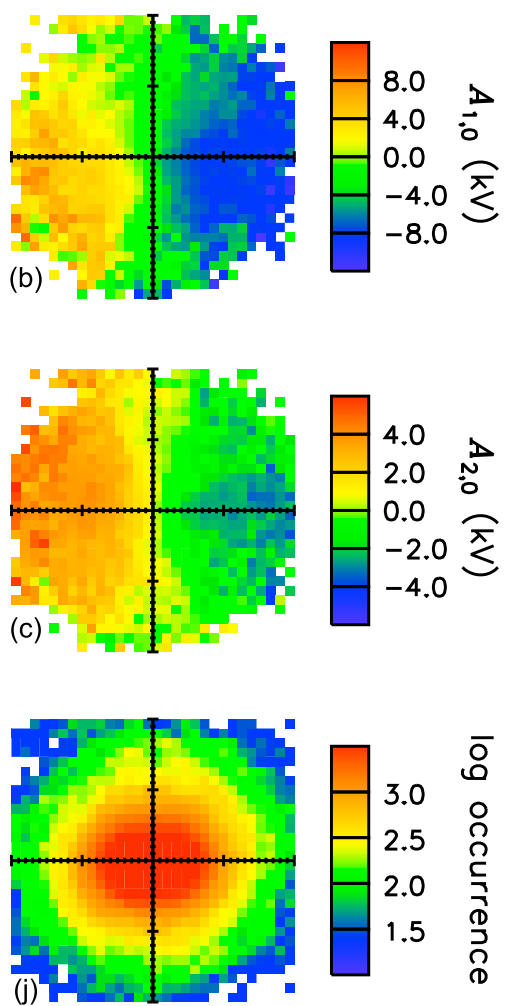
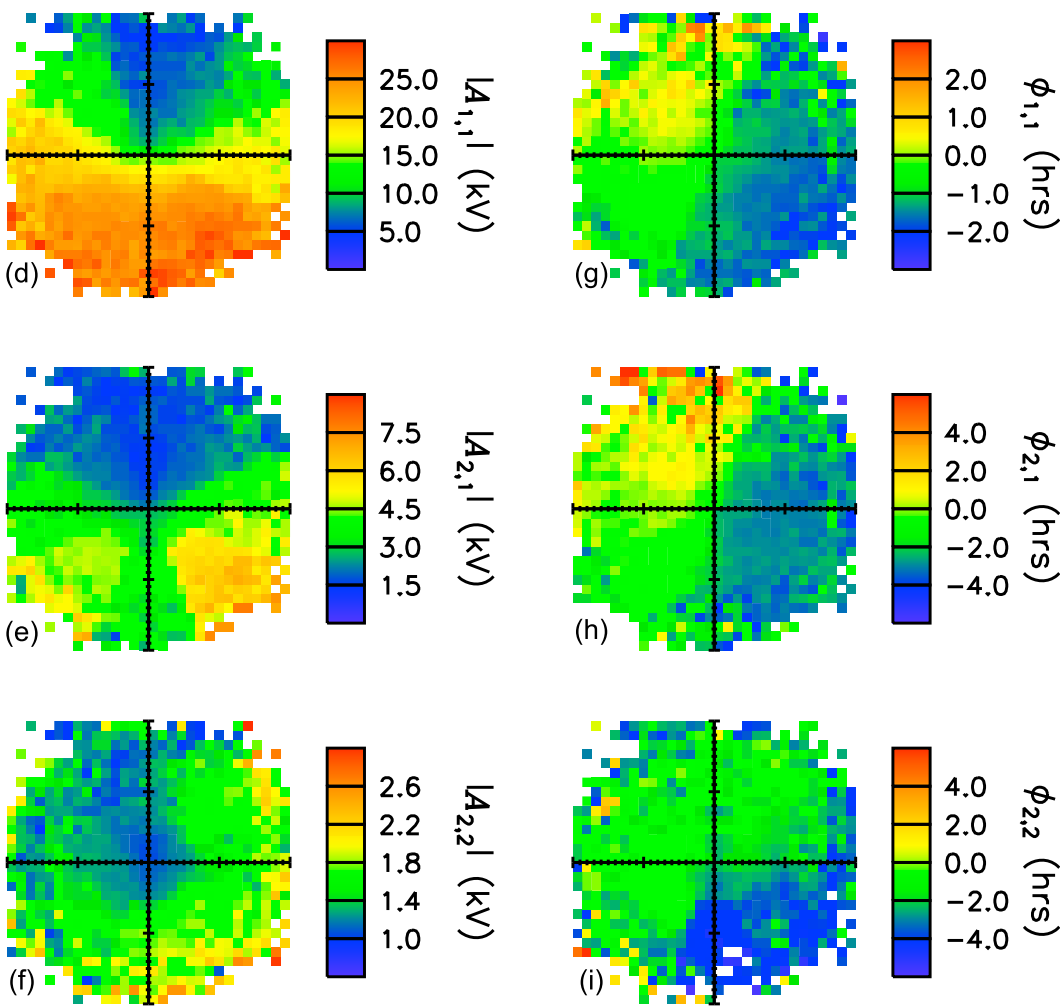

Figure 6. (a-i) Distributions of mean values of the first six coefficients, binned according to corresponding values of IMF $B_{Y}$ (left to right) -20 to $+20 \mathrm{n}$ and $B_{Z}$ (top to bottom) +20 to $-20 \mathrm{nT}$. (j) Occurrence distribution of mean values. The $A_{l, 0}$ coefficients are shown in Figures $6 \mathrm{a}-6 \mathrm{c}$. The amplitude of the $A_{1,1}, A_{2,1}$, and $A_{2,2}$ coefficients are shown in Figures $6 \mathrm{~d}-6 \mathrm{f}$, and their rotation angles are shown in Figures $6 \mathrm{~g}-6 \mathrm{i}$. The data in each plot are scaled according to the corresponding color bar on the right.

coefficients is shown. In many cases the distributions within each bin are approximately Gaussian and thus the mean provides a suitable indication of the general dependence on the IMF. In some bins the distributions are clearly nonGaussian, and we investigate these instances further below. To provide a simple measure of the variability within each bin, their standard deviations are presented in Figure 7 , in the same format as Figure 6 . In both figures the $A_{l, 0}$ coefficients are shown in Figures $6 \mathrm{a}-6 \mathrm{c}$ and $7 \mathrm{a}-7 \mathrm{c}$, with the amplitude and rotation of the $A_{1,1}, A_{2,1}$, and $A_{2,1}$ coefficients shown in Figures $6 \mathrm{~d}-6 \mathrm{f}$ and $7 \mathrm{~d}-7 \mathrm{f}$ and Figures $6 \mathrm{~g}-6 \mathrm{i}$ and $7 \mathrm{~g}-7 \mathrm{i}$, respectively. In Figures 6 and 7 the data in each plot are scaled according to the corresponding color bar on the right. In Figure 6 the range of each color bar was chosen based on the corresponding distribution shown in Figure 4.
In Figure 7 the color bars are scaled to the magnitudes of the corresponding ranges from Figure 6.

[21] Consider first the $A_{l, 0}$ coefficients, shown in Figures $6 \mathrm{a}-6 \mathrm{c}$, which all show some degree of dependence on IMF $B_{Y}$. The strong negative bias in the $A_{0,0}$ coefficient (discussed in section 4.1) is evident, with only the $B_{Y}<0, B_{Z}<0$ quadrant containing a significant fraction of positive values. This implies that only under strongly driven negative IMF $B_{Y}$ conditions does the positive potential (dawn) cell dominate. The standard deviations, $\sigma$, of these mean values, shown in Figure $7 \mathrm{a}$, are $\sim 3-6 \mathrm{kV}$, suggesting some degree of variability and a correlation analysis of $A_{0,0}$ with IMF $B_{Y}$ reveals a weak linearity with correlation coefficient, $r=-0.35$. The $A_{1,0}$ and $A_{2,0}$ coefficients exhibit a clearer association with $B_{Y}$. For $A_{1,0}, \sigma \approx 4 \mathrm{kV}$ and $r=-0.63$ and for $A_{2,0}, \sigma \approx 2 \mathrm{kV}$ and $r=-0.67$. These coefficients 

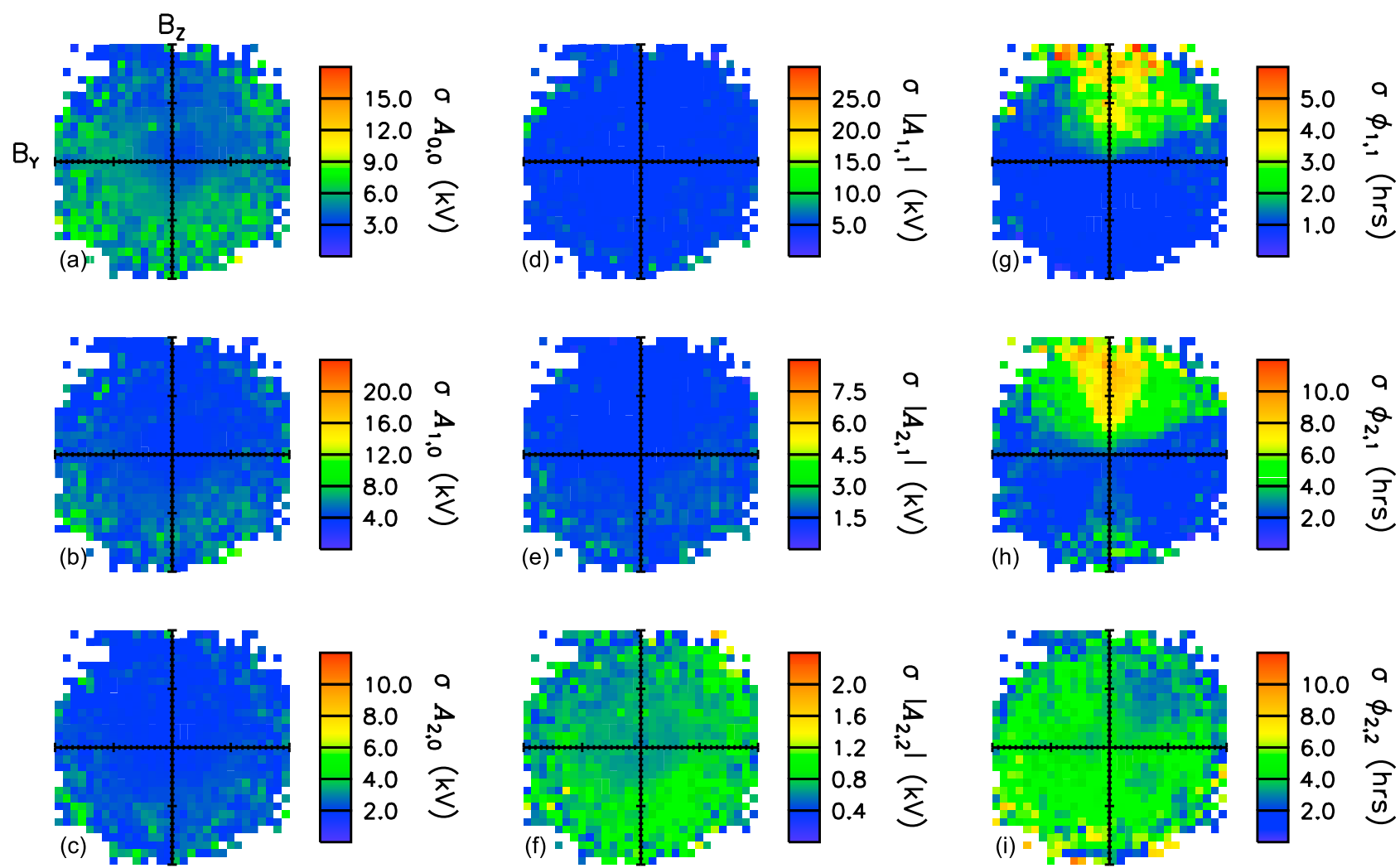

Figure 7. Standard deviations of the mean values of the first six coefficients plotted versus IMF $B_{Y}$ and $B_{Z}$, shown in the same format as Figures $6 \mathrm{a}-6 \mathrm{i}$.

appear to have a dominant role in describing IMF $B_{Y}$ asymmetries in the potential patterns and are discussed further in section 5 .

[22] Next, we consider the amplitude of the $A_{1,1}$ coefficients, shown in Figure 6d, which govern the strength of the dominant twin vortex component of the convection pattern. As expected, $\left|A_{1,1}\right|$ displays a clear dependence on IMF $B_{Z}$, becoming larger as $B_{Z}$ decreases from positive through to negative values. A modest asymmetry about $B_{Z}=0$ suggests that negative $B_{Y}$ is slightly more efficient at driving this mode of convection, evidenced by the fact that for negative $B_{Y}\left|A_{1,1}\right|$ increases more rapidly with decreasing $B_{Z}$ than for positive $B_{Y}$. Correlation analysis reveals a strong linear dependence of $\left|A_{1,1}\right|$ on $B_{Z}$ with $r=-0.66$, which is supported by the generally low standard deviation of $\sigma<5 \mathrm{kV}$.

[23] A slightly more complicated relationship exists for $\left|A_{2,1}\right|$ shown in Figure 6e. A similar general dependence on IMF $B_{Z}$ exists, with small amplitudes for positive $B_{Z}$ and larger amplitudes for negative $B_{Z}$. However, the largest amplitudes correspond to where $B_{Z}=-\left|B_{Y}\right|$, i.e., where the magnitude of the IMF clock angle is $135^{\circ}$. This suggests that the $A_{2,1}$ coefficient predominantly describes IMF $B_{Y}$ associated asymmetries in the potential pattern, but only those that appear in the presence of strong twin vortex convection. $\left|A_{2,1}\right|$ is particularly high for positive $B_{Y}$; the significance of this is discussed further in section 5.1. It is also worth noting here that for this coefficient in particular, there appears to be some evidence for the IMF binning of the model vectors used in the electric potential fitting described in section 3 . The Ruohoniemi and Greenwald [1996] model patterns were classified by IMF clock angle in bins of $45^{\circ}$ which appear to be manifest as sharp transitions between similar clock angle regimes in the distribution of $\left|A_{2,1}\right|$ (and to a lesser extent in some of the other distributions). This effect can be reduced by increasing the minimum threshold of $N$ discussed above, but this then limits the statistics available for the analysis. As we are primarily interested in IMF related trends we do not consider it problematic that this effect exists and, in fact, we later show that where significant variability exists within one of the model bins the data are sufficient to dominate the resulting patterns. In future studies, if such IMF binning of the model data proved problematic, it could be worth investigating the use of a dynamical model such as that described by Cousins and Shepherd [2010].

[24] The remaining coefficient shown in Figure 6 is $A_{2,2}$. In this case the amplitude exhibits only a weak IMF dependence being generally larger for positive IMF $B_{Y}$ and for negative $B_{Z}$, but also being generally larger for higher magnitude IMF values, irrespective of their direction. An exception to this appears to be for positive $B_{Z}$ and weakly negative $B_{Y}$ where low amplitudes seem to dominate throughout. Correlation analysis therefore suggests that no simple linear relationship exists, with the highest correlation of $\mathrm{r}=0.18$ existing between $\left|A_{2,2}\right|$ and $\sqrt{B_{Y}^{2}+B_{Z}^{2}}$. Inspection of the corresponding standard deviations also suggests somewhat higher variability, consistent with only a weak IMF dependence.

[25] Lastly, we turn to the right-hand columns of Figures 6 and 7, which illustrate the relationship between the IMF and the rotation of the basis functions given by $\phi_{l m}$. An initial 

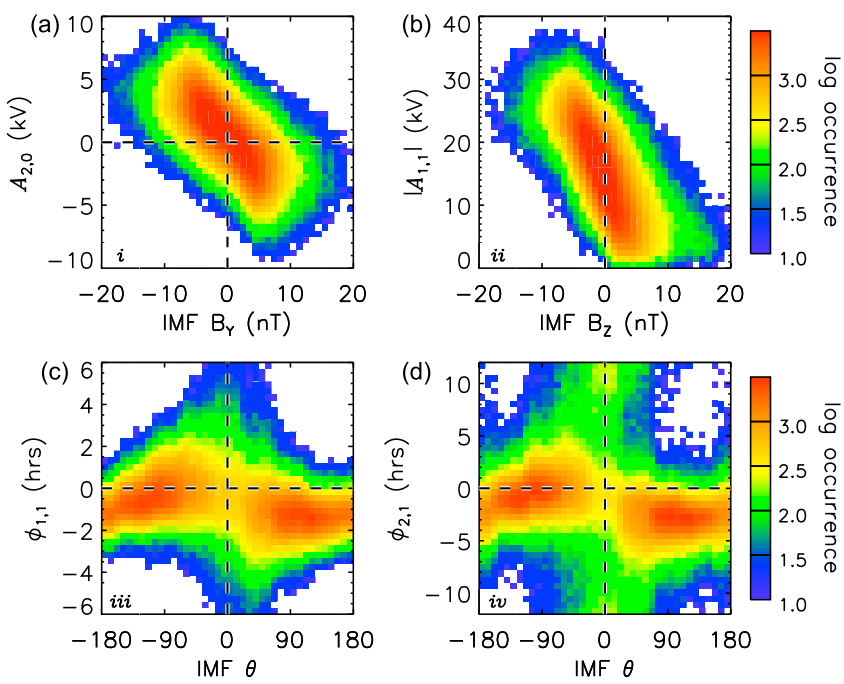

Figure 8. Coefficient occurrence distributions for (a) IMF $B_{Y}$ versus $A_{2,0}$, (b) IMF $B_{Z}$ versus $\left|A_{1,1}\right|$, (c) IMF $\theta$ versus $\phi_{1,1}$, and (d) IMF $\theta$ versus $\phi_{2,1}$. The occurrence is shown on a logarithmic scale indicated by the color bars on the right.

inspection suggests similar distributions for $\phi_{1,1}$ and $\phi_{2,1}$, shown in Figures $6 \mathrm{~g}$ and $6 \mathrm{~h}$. Both show a modest negative bias (clockwise rotation), which is strongest for $B_{Y}>0$, and are only positive (anticlockwise rotation) in the $B_{Y}<0, B_{Z}>0 \mathrm{IMF}$ quadrant. This reveals an interesting contrast to the IMF $B_{Y}$ dependence of the $A_{0,0}$ coefficients. As discussed above, $A_{0,0}$ introduces a positive bias in the potential only for strongly driven (i.e., $B_{Z}<0$ ), $B_{Y}<0$ conditions. The rotation of the pattern, on the other hand, is only positive for weakly driven (i.e., $B_{Z}>0$ ), $B_{Y}<0$ conditions. This is considered further in section 5.2. It is also significant to note the different scales used for these two distributions. Both represent $m=1$ basis functions and therefore have $24 \mathrm{~h}$ rotational symmetry yet we have only shown a limited range of $\phi_{l m}$ in each case $(-3$ to $+3 \mathrm{~h}$ for $\phi_{1,1}$ and -6 to $+6 \mathrm{~h}$ for $\left.\phi_{2,1}\right)$, based on the limited ranges implied by their respective occurrence distributions shown in Figure 4. However, both of these distributions actually have significant non-Gaussian tails which result in the mean values presented here misrepresenting real populations within the distributions. This is evidenced by the distributions of standard deviations for these coefficients, which reveal considerable variability in particular for strongly northward IMF conditions, as shown in Figures $7 \mathrm{~g}$ and $7 \mathrm{~h}$. We consider this further in section 5.1. First, we briefly mention the $\phi_{2,2}$ distributions shown in Figures $6 \mathrm{i}$ and $7 \mathrm{i}$. These also exhibit some dependence on the IMF evidenced by the large negative rotation under $B_{Z}<0, B_{Y}>0$ conditions. However, like $\left|A_{2,2}\right|$, the standard deviations of these values are relatively high, suggestive of a much weaker relationship and resultant non-Gaussian distribution.

[26] The data presented above reveal two categories of IMF dependence of the coefficients. In some cases a set of reasonably narrow Gaussian distributions of the coefficients exists, which show a clear and often linear dependence on one or both IMF components. In other cases, non-Gaussian elements of the distributions reveal a more complex, nonlinear relationship with the IMF. Examples of these two categories are presented in Figure 8, which shows coefficient occurrence distributions for IMF $B_{Y}$ versus $A_{2,0}$ (Figure 8a), IMF $B_{Z}$ versus $\left|A_{1,1}\right|$ (Figure $8 \mathrm{~b}$ ), IMF $\theta$ versus $\phi_{1,1}$ (Figure $8 \mathrm{c}$ ), and IMF $\theta$ versus $\phi_{2,1}$ (Figure $8 \mathrm{~d}$ ). In each case the occurrence is shown on a logarithmic scale from blue to red, as indicated by the color bar on the right, to emphasize some of the lowoccurrence regions, as discussed below.

[27] The first two plots reveal the clear linear dependencies of $A_{2,0}$ and $\left|A_{1,1}\right|$ on IMF $B_{Y}$ and $B_{Z}$, respectively. These dependencies are relatively straightforward to explain with reference to Figure 2. As discussed in section 4.1 the $A_{1,1}$ coefficient describes the basic twin vortex potential distribution, the magnitude of which has a well established dependence on IMF $B_{Z} \cdot A_{2,0}$, on the other hand, introduces a circularly symmetric component to the potential distribution, strengthening the dusk cell at latitudes where it weakens the dawn cell, and vice versa. The net result of this is to introduce a dusk-dawn asymmetry to the preexisting twin vortex pattern, an effect well established to be associated with IMF $B_{Y}$. The combination of different basis functions in this way is considered further in section 5.1.

[28] The third and fourth plots of Figure 8 reveal the somewhat more complex dependencies of $\phi_{1,1}$ and $\phi_{2,1}$ on the IMF clock angle, $\theta$. In both cases the overall bias of $\phi_{l m}$ toward negative values is evident, indicative of the tendency for the convection in the polar cap to be oriented slightly away from the noon-midnight meridian. The general tendency for $\phi_{l m}$ to be positive for only small negative clock angles, as discussed above in reference to Figure 6, is also apparent. This phenomenon is discussed further in section 5.2. There is also a stretching of the distributions toward larger $\phi_{l m}$ at small clock angles, that is particularly evident for $\phi_{2,1}$. In this case, a small but clear secondary peak in the distribution exists for $-11 \mathrm{~h}>\phi_{2,1}>9 \mathrm{~h},|\theta|<10^{\circ}$. The significance of this peak is discussed further in section 5.1.

\section{Discussion}

[29] In section 4 we have reported the fundamental properties of the coefficients of a spherical harmonic expansion of the ionospheric electric potential. By identifying the coefficients associated with specific electric potential (or associated convection) characteristics it is possible to derive statistics about the convection and its relationship to possible driving mechanisms, such as coupling with the IMF. In this section we consider some of the applications of this analysis in developing more quantitative techniques for investigating asymmetries in the convection pattern.

\subsection{Identifying Convection Characteristics}

[30] To help illustrate our discussion in this section we provide in Figure 9 three examples of how the basis functions in Figure 2 combine to build up different characteristic convection patterns. In each of the three cases, four stages of the 'build' are shown (from left to right), with the amplitude and/or rotation of the added basis function detailed in each case. Only very low order functions are included, and the patterns are not meant to represent real observations, but simply be illustrative of the basic shapes associated with different IMF conditions. The specific values of each coefficient were chosen, by eye, from inspection of the data in 

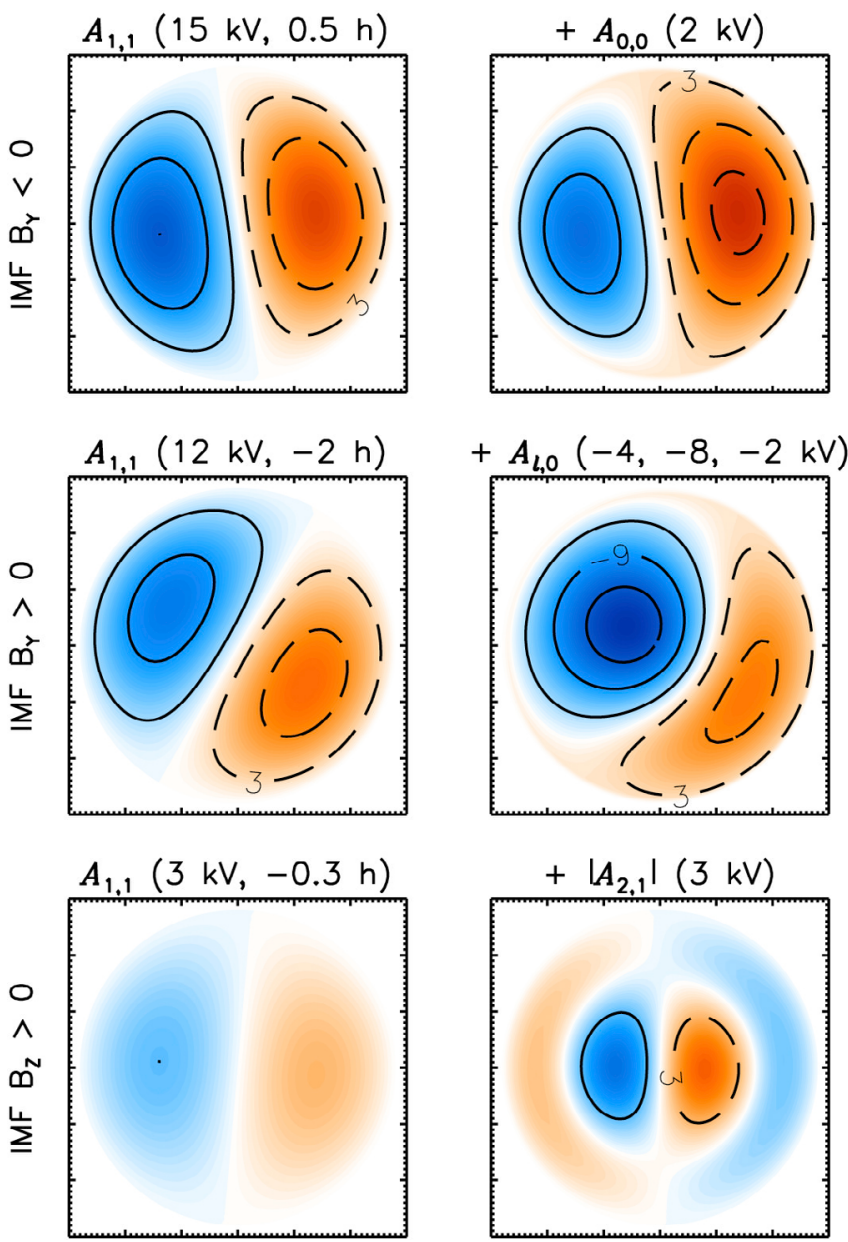
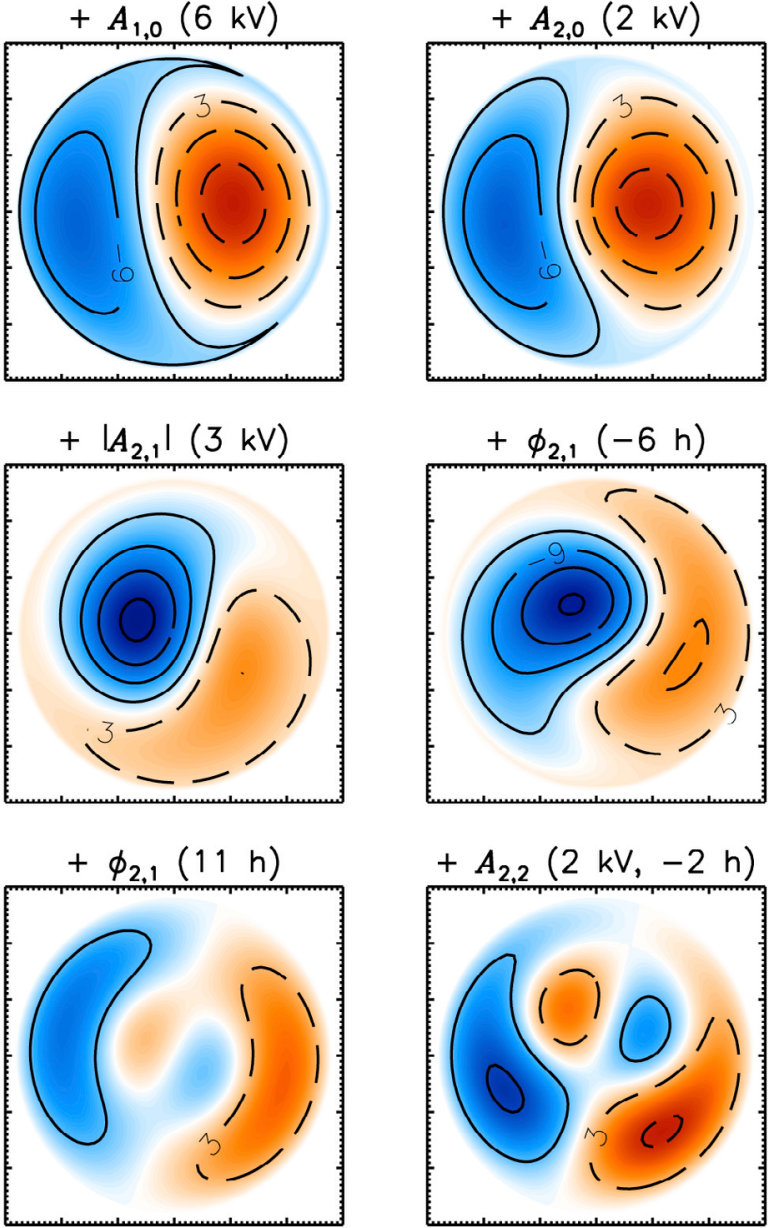

Figure 9. An illustration of the way in which the different order basis functions combine to produce the characteristic convection pattern shapes associated with different orientations of IMF. The specific values of each coefficient are given in parentheses and are discussed in the text.

Figure 6. The first row illustrates a simple $B_{Y}<0, B_{Z}<0$ scenario. As discussed above, the $m=0$ coefficients are largely responsible for the large-scale $B_{Y}$ asymmetry and as shown here, a quite realistic convection pattern need only contain the first 3 order $m=0$ coefficients in addition to the basic twin vortex $A_{1,1}$ basis function. Indeed, as indicated by the relative locations of the triangle and square symbols in Figure 4 , the IMF $B_{Y}<0$ convection pattern tends to have generally lower amplitude contributions from most coefficients (with the exception of $\left|A_{1,1}\right|$ ) and rotation angles closer to zero (or $12 / \mathrm{m}$, where appropriate). This implies that for $B_{Y}<0$, the convection pattern is closer to the generic twin vortex convection pattern than is the case for $B_{Y}>0$.

[31] The second row illustrates the components of a $B_{Y}>0, B_{Z}<0$ pattern. We begin with a twin vortex, rotated into a more typical orientation $\left(\phi_{1,1}=-2 \mathrm{~h}\right)$, and then add in the first three $A_{l, 0}$ components at once (corresponding, in this case, to the three values given in parentheses in the figure). Inspection of Figure $3 \mathrm{~d}$ reveals this to be a more complex pattern than the $B_{Y}<0$ case, and Figure 6 e suggests that the $A_{2,1}$ basis function may be largely responsible. Figure $6 \mathrm{~h}$ indicates a significant rotation of this function and so, for clarity, we illustrate the effects of $\left|A_{2,1}\right|(3 \mathrm{kV})$ and $\phi_{2,1}(-6 \mathrm{~h})$ separately. Again, we stress that the resulting pattern does not necessarily represent a real example, but the key features differentiating this pattern from the $B_{Y}<0$ one are clear. Most notable, are the 'tails' on the twin cells, which we suggest (in the case of the nightside 'tail') may be a manifestation of the Harang reversal [Heppner, 1972; Hughes and Bristow, 2003], in which the dusk convection cell reverses direction from east to west in the midnight sector. As discussed by Grocott et al. [2010], while this feature is related to substorm dynamics and may therefore be present under either orientation of IMF $B_{Y}$, it is more evident for $B_{Y}>0$. This is simply because the $B_{Y}$ controlled element of the nightside auroral zone pattern is opposite to the Harang element in this case, producing the observed double west-east-west reversal associated with the 'tail' described here. Grocott et al. [2010] drew qualitative conclusions regarding this phenomenon, however, the globally descriptive nature of the coefficients would make possible future quantitative analyses of the relative control of IMF $B_{Y}$ and the substorm electrodynamics in controlling the evolution of the convection pattern.

[32] In the bottom row of Figure 9 we illustrate one possible case of IMF $B_{Z}>0$ convection. We stress that this is only one possible case since, as discussed in section 4.2, there are multiple peaks at zero IMF clock angle in some of 
the coefficient distributions (e.g., the $A_{2,1}$ distribution shown in Figure 8d) implying a range of possible convection patterns under these conditions. In this case, we begin with a very weak twin vortex pattern $\left(\left|A_{1,1}\right|=3 \mathrm{kV}\right)$ and add a comparable contribution from $\left|A_{2,1}\right|$. We then chose a value of $\phi_{2,1}=11 \mathrm{~h}$, corresponding to the peak at $\sim 11 \mathrm{~h}$ in the occurrence distribution shown in Figure 8d, before adding in a component from $A_{2,2}$ to produce a 4 cell pattern that includes both a low-latitude convection cell pair and a highlatitude reverse convection cell pair. Such convection has previously been observed under strongly northward IMF by, e.g., Imber et al. [2006, 2007], although it is important to note that such clearly defined twin reversed convection cells are not often observed. This can be seen in, for example, the statistical study of Ruohoniemi and Greenwald [2005, Figures 5, 6, and 7] which demonstrate that even for strong northward IMF the average convection pattern only contains weak evidence for twin reverse cells. This can be explained by the significant spread in the occurrence distribution of, e.g., $\phi_{2,1}$ shown in Figure $8 d$, which suggests that different convection characteristics can exist for similar instantaneous IMF conditions, particularly in the strongly northward case. We suggest that isolating the basis functions responsible for describing a given characteristic pattern, or element, of convection, may be a more appropriate method of studying the phenomenology than the use of a simple IMF classification.

[33] To demonstrate this, we present in Figure 10 the occurrence distributions of a subset of $A_{l m}$, presented in the same format as Figure 4. In this case, only those intervals identified by eye as containing clear evidence for reverse twin cell convection, from a manual survey of 1 year of data from December 2000 to December 2001, have been included. This somewhat laborious and subjective process yielded 219 intervals for which we also show the distribution of IMF $B_{Y}$ and $B_{Z}$ in the inset plot. The data in this plot reveal a clear demarcation of IMF values under which such convection patterns can occur $(89 \%$ of intervals lie within the region indicated by the solid lines) but these represent fewer than $1 \%$ of all the patterns from this 1 year period that were observed during intervals of similar IMF orientation, reaffirming our earlier claim that a variety of convection scenarios must exists for such conditions. Turning to the distributions of the coefficients, although some spread is evident - especially in $\phi_{l m}$ - there are clear peaks in all of the distributions which, in most cases, align well with the location of the diamond symbol representing the example $B_{Z}+/$ twin reverse cell pattern from Figure 3 . It is clear, therefore, that a much more efficient and objective method for identifying such characteristic flows is to define selection criteria based on the roles of the various basis functions.

\subsection{Quantifying Convection Characteristics}

[34] Although many of the features and relationships discussed above are evident, visually, in the convection patterns of, e.g., Ruohoniemi and Greenwald [2005], utilization of the appropriate coefficients enables the morphology of the convection pattern to be studied in a purely quantitative way. For example, we mentioned above that $\Phi_{\text {diff }}$ is often used to study the strength of the convection. A simple analysis of, e.g., the relationship between $\Phi_{\text {diff }}$ and IMF $B_{Z}$ reveals a modest dependence with a correlation coefficient of $|r|=0.60$, which is actually lower than the value of 0.67 quoted in section 4.2 for the correlation of $\left|A_{1,1}\right|$ and $B_{Z}$. This could be explained by the existence of additional driving mechanisms unrelated to IMF $B_{Z}$, such as viscous interactions [Axford and Hines, 1961] for example, which might contribute to $\Phi_{\text {diff }}$ via different modes of convection. It could also be related to time-dependent phenomena such as substorms, which may contribute to $\Phi_{\text {diff }}$ but whose occurrence will not necessarily correlate with the instantaneous value of $B_{Z}$. We also introduced $\Phi_{\text {res }}$, which provides a measure of asymmetry in the potential. This asymmetry, however, appears to be only weakly associated with IMF $B_{Y}$; inspection of the data suggests a weak dependence with correlation coefficient $|r|=0.21 . A_{2,0}$, on the other hand, is correlated much more strongly with $B_{Y}(|r|=0.67)$. In other words, by isolating individual components of the convection pattern, in terms of their associated basis functions, we may gain a better understanding of the driving mechanisms involved than is possible using traditional measures alone.

[35] To illustrate this further, we consider again the existence of two separate manifestations of IMF $B_{Y}$ asymmetry in the convection patterns discussed in section 4.2. One is the asymmetry associated with $A_{0,0}$ and the other is the rotation of the first order pattern, described by $\phi_{1,1}$. We noted in section 4.2 that $A_{0,0}$ introduces a positive bias in the potential only for strongly driven (i.e., $B_{Z}<0$ ), $B_{Y}<0$ conditions, whereas $\phi_{1,1}$ is only positive for weakly driven (i.e., $\left.B_{Z}>0\right), B_{Y}<0$ conditions. To provide a simple comparison of the relative contribution of $A_{0,0}$ and $\phi_{1,1}$ we define $R$ :

$$
R=\frac{\left|\Delta \phi_{1,1}\right|-\left|\Delta A_{0,0}\right|}{\| \Delta \phi_{1,1}|-| \Delta A_{0,0} \mid}\left|\frac{\Delta \phi_{1,1}}{\max \left|\Delta \phi_{1,1}\right|}-\frac{\Delta A_{0,0}}{\max \left|\Delta A_{0,0}\right|}\right|
$$

where $\Delta \phi_{1,1}$ and $\Delta A_{0,0}$ indicate perturbations of $\phi_{1,1}$ and $A_{0,0}$ from their respective median values. The magnitude of $R$ therefore represents the absolute relative difference between $\Delta \phi_{1,1}$ and $\Delta A_{0,0}$, with the sign of $R$ being positive for $\left|\Delta \phi_{1,1}\right|>\left|\Delta A_{0,0}\right|$ and negative for $\left|\Delta \phi_{1,1}\right|<\left|\Delta A_{0,0}\right|$.

[36] In Figures 11a and 11b we present the distributions of the mean and the standard deviation of $R$, respectively, binned according to corresponding values of IMF $B_{Y}$ and $B_{Z}$ in a similar format to Figures 6 and 7. For more strongly driven $\left(B_{Z}<0\right)$ conditions, $R$ tends to be negative, whereas for more weakly driven $\left(B_{Z}>0\right)$ conditions $R$ tends to be positive. The implications of this are that for strongly driven conditions the dominant asymmetry is the offset between the positive and negative potential cells. This is evidenced by the fact that as $B_{Y}$ becomes more strongly negative (with $B_{Z}$ also negative) $R$ decreases from $\sim-0.1$ to $\sim-0.3$. For more weakly driven conditions the dominant asymmetry is the rotation of the pattern. This is evidenced by the fact that as $B_{Z}$ becomes more positive, $R$ increases from $\sim 0$ to $\sim+0.3$. We suggest an interpretation of this in terms of balancing the azimuthal magnetic tension force, imparted on open field lines by IMF $B_{Y}$ and acting to cause a rotation of the pattern, with the required change in momentum of the plasma frozen in to the magnetic flux being otherwise carried antisunward with the solarwind. If the rate of antisunward flux transport is large, as expected for $B_{Z}<0$, then the force required to rotate the associated momentum vector is also large in comparison to the magnetic tension force and so 

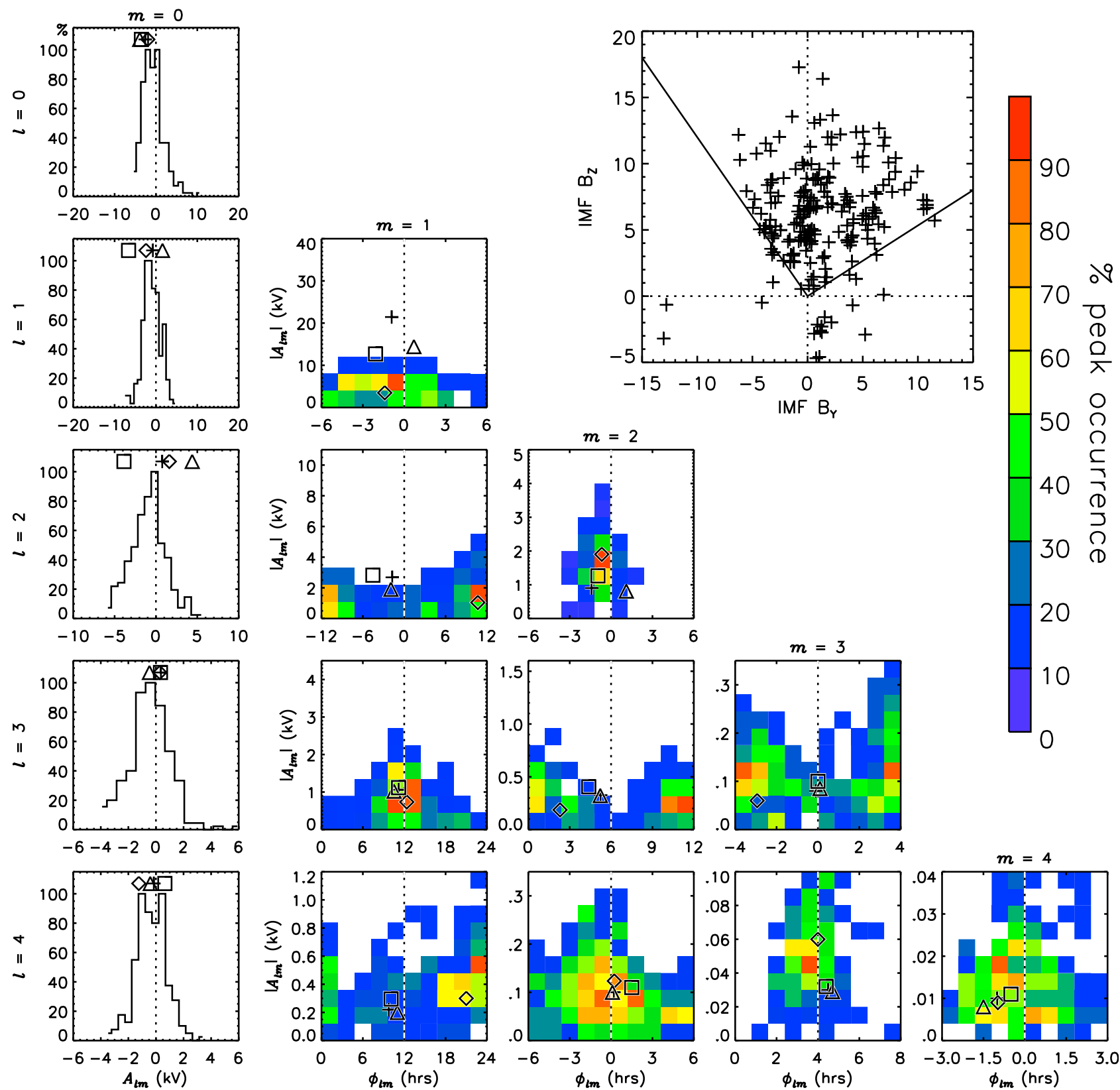

Figure 10. Occurrence distributions of a subset of $A_{l m}$ presented in the same format as Figure 4 . In this case, only those intervals identified by eye as containing clear evidence for reverse twin cell convection, from a manual survey of 1 year of data from December 2000 to December 2001, have been included. The distribution of IMF $B_{Y}$ and $B_{Z}$ for these intervals is shown in the additional plot, with the approximate limits of $B_{Y}$ and $B_{Z}$ indicated by the solid lines.

little rotation is observed. If the rate of antisunward flux transport is low, then the same magnetic tension force imparts a greater degree of rotation. A thorough investigation of this hypothesis is beyond the scope of the present paper but it nevertheless serves to illustrate the possible applications of this kind of analysis.

[37] Our discussion thus far has focused on statistical analyses, however, analysis of the coefficients is equally appropriate to case studies. We illustrate this with the example time series of data from 08:00-18:30 UT on 12 March 2001 shown in Figure 12. The top two plots show the IMF $B_{Z}$ and $B_{Y}$ components, which can be loosely characterized in terms of three subintervals: a, $B_{Z}<0$, $B_{Y}>0 ; \mathrm{b}, B_{Z}>0, B_{Y}>0$; and c, $B_{Z}<0, B_{Y}<0$. The vertical dashed lines indicate representative times for each subinterval, for which the electric potential patterns are presented in Figure 13. The remaining six plots of Figure 12 show a selection of coefficient parameters, which evolve with the changing IMF, contributing to the three example patterns at the times shown. $A_{1,0}$ and $A_{2,0}$ essentially mirror $B_{Y}$, illustrating their dominant role in describing the $B_{Y}$ effect in the potential patterns. The change in these coefficients between lines a and $\mathrm{c}$, along with the modest net change also evident in $\phi_{1,1}$ and $\phi_{2,1}$, are responsible for the primary differences 

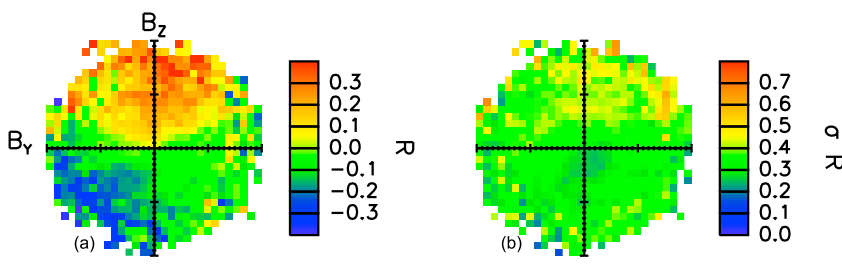

Figure 11. Distributions of (a) mean values and (b) standard deviations of the mean of the quantity $R$ (defined in equation (4)), binned according to corresponding values of IMF $B_{Y}$ (left to right) -20 to $+20 \mathrm{nT}$ and $B_{Z}$ (top to bottom) +20 to $-20 \mathrm{nT}$. Each plot is color coded according to the bar on the right.

evident between the patterns in Figures $13 \mathrm{a}$ and $13 \mathrm{~b} .\left|A_{1,1}\right|$ more closely mirrors $B_{Z}$, being essentially the same at lines $\mathrm{a}$ and $\mathrm{c}$ but dropping significantly at line $\mathrm{b}$. This time coincides with a local maximum in $\left|A_{2,2}\right|$ and a large rotation in $\phi_{2,1}$ by $\sim 12 \mathrm{~h}$ which together are largely responsible for the multicelled pattern shown in Figure 13b. This pattern actually consists of 3 distinct cells, rather than 4 (as in Figure $3 \mathrm{~b}$ ), and could represent a transitory stage during the evolution from 2 to 4 cells, or be a result of insufficient data coverage. Either way, the 'reversed' sunward convection discussed above is still clearly evident, thus demonstrating the coefficients to be a robust method of determining key convection characteristics. Ultimately the choice, and interpretation, of coefficients for any given study will be governed by the nature of the phenomenon under investigation.

[38] Lastly, in addition to using the coefficients to study characteristic features in the convection, they could also be used for data validation. For any given case, the coefficients could be compared to the statistical distributions to determine whether they are likely to represent a characteristic pattern or simply an example of noisy data or a poor fit. In a similar way to a case of unfeasibly high transpolar voltage leading one to treat a convection pattern as being suspect, for example, so too could unlikely combinations of convection pattern coefficients.

\section{Summary}

[39] We have discussed a method of utilizing spherical harmonic coefficients derived from an established SuperDARN convection mapping analysis technique to describe different characteristic patterns of ionospheric convection and their relation to upstream interplanetary magnetic field conditions and magnetospheric dynamics. In particular, we have used the large-scale IMF clock angle dependence to illustrate our technique, however, the scope exists for a wealth of different convection characteristics to be investigated. Each basis function of the spherical harmonic expansion describes a different component of the pattern and so, by isolating the functions responsible for describing any particular component, the shape, as well as the strength of the convection can be quantitatively studied. In this paper we have outlined some of the basic convection characteristics and their association with various basis functions. In addition, we have quantitatively compared the coefficients with concurrent IMF data to elucidate the nature of the relationship between
IMF $B_{Y}$ and $B_{Z}$ and the shape and strength of the convection. Our main findings can be summarized as follows:

[40] 1. The strength of the twin vortex flow is described by $\left|A_{1,1}\right|$, which is highly correlated with $\Phi_{\text {diff }}(r=0.92)$ and strongly dependent on IMF $B_{Z}(r=-0.66)$.

[41] 2. A (generally westward) rotation of the convection pattern is described by $\phi_{1,1}$ (with a modal value of $\sim-1 \mathrm{~h}$ ) and is weakly dependent on $\operatorname{IMF} B_{Y}(r=0.30)$. A maximum correlation of $r=0.43$ is found if only IMF clock angles of $|\theta|>68^{\circ}$ are considered.

[42] 3. A tendency for the dusk (negative potential) convection cell to be stronger is described by $A_{0,0}$ (having modal value of $-3.66 \mathrm{kV}$ ).

[43] 4. The dusk-dawn asymmetry of the twin vortex pattern is (largely) described by $A_{1,0}$ and $A_{2,0}$, which are strongly dependent on IMF $B_{Y}(r=-0.63$ and $r=-0.67$, respectively).

[44] 5. Additional asymmetries that appear to be associated with the Harang Discontinuity, are described by $A_{2,1}$.

[45] 6. 'Reverse' flow high-latitude convection cells are described by $\phi_{2,1} \sim 12 \mathrm{~h}$ and elevated $\left|A_{2,2}\right|$.

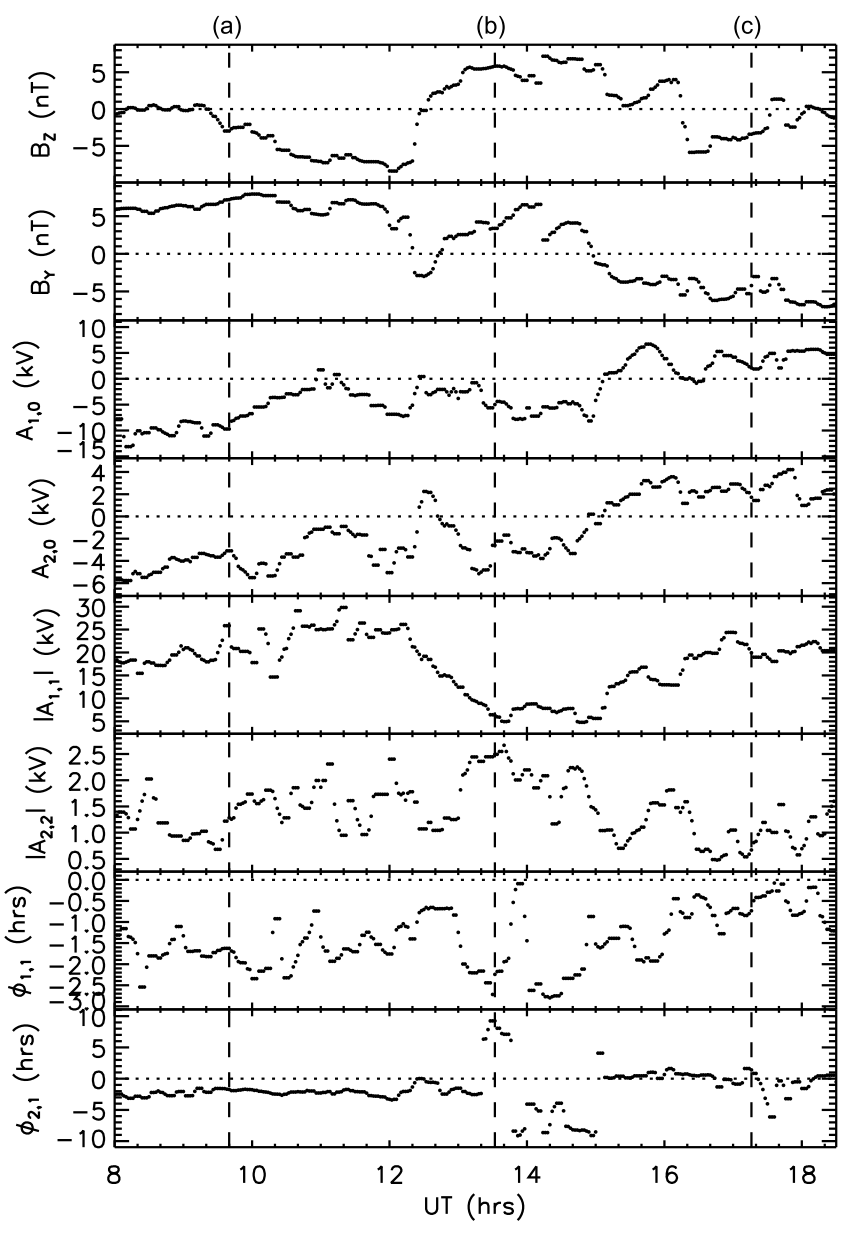

Figure 12. Time series of data from 08:00 to 18:30 UT on 12 March 2001. IMF $B_{Z}$ and $B_{Y}$ are plotted in the top two panels with selected coefficient parameters shown in subsequent panels. The vertical dashed lines indicate times of interest described in the text, corresponding to the times of the electric potential patterns shown in Figure 13. 
(a)

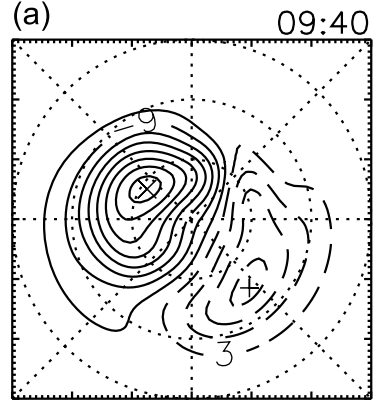

(b)

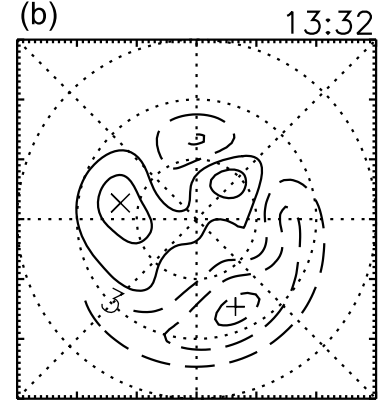

(c)

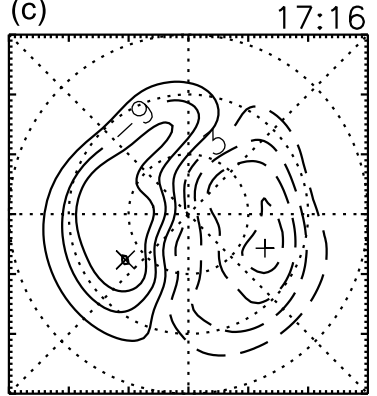

Figure 13. Three electric potential patterns, from (a) 09:40, (b) 13:32, and (c) 17:16 UT on 12 March 2001, shown in magnetic local time, magnetic latitude coordinates. In each plot, noon is to the top and dusk to the left. The concentric dashed circles represent $10^{\circ}$ increments of magnetic latitude, marked at $60^{\circ}, 70^{\circ}$, and $80^{\circ}$.

[46] Our results indicate that the spherical harmonic coefficients provide a convenient and suitable framework in which to define the different characteristic patterns of convection and a valid measure of the degree to which external influences, such as the IMF orientation, affect the convection pattern shape. Our list of conclusions is not exhaustive but is merely exemplary of the phenomenology that could be investigated using this technique.

[47] Acknowledgments. We would like to thank the PIs of the SuperDARN radars for provision of the radar data employed in this study. The data employed were from radars funded by the research agencies of Japan, Canada, the U.S., France, and the U.K. We also thank R. J. Barnes of the Johns Hopkins University for the SuperDARN analysis software. A.G. and S.E.M. were supported during this study by NERC grant NE/ G019665/1. S.M.I. was supported by EU grant FP7-SPACE-2010-1263325. S.E.M., M.L., and T.K.Y. were also supported by STFC grant $\mathrm{ST} / \mathrm{H} 002480 / 1$

[48] Robert Lysak thanks the reviewers for their assistance in evaluating this paper.

\section{References}

Axford, W. I., and C. O. Hines (1961), A unifying theory of high-latitude geophysical phenomena and geomagnetic storms, Can. J. Phys., 39(10), 1433-1464, doi:10.1139/p61-172.

Baker, J. B. H., and R. A. Greenwald (2003), Eigenmode analysis of IMF-dependent ionospheric convection as derived from the TIMED/ SuperDARN database, Geophys. Res. Abstr., 5, Abstract 14397.

Baker, J. B. H., A. J. Ridley, V. O. Papitashvili, and C. R. Clauer (2003), The dependence of winter aurora on interplanetary parameters, J. Geophys. Res., 108(A4), 8009, doi:10.1029/2002JA009352.

Chisham, G., et al. (2007), A decade of the Super Dual Auroral Radar Network (SuperDARN): Scientific achievements, new techniques and future directions, Surv. Geophys., 28, 33-109, doi:10.1007/s10712007-9017-8.

Cousins, E. D. P., and S. G. Shepherd (2010), A dynamical model of highlatitude convection derived from SuperDarn plasma drift measurements, J. Geophys. Res., 115, A12329, doi:10.1029/2010JA016017.

Greenwald, R. A., et al. (1995), Darn/SuperDarn: A global view of the dynamics of high-latitude convection, Space Sci. Rev., 71, 761-796, doi:10.1007/BF00751350.

Grocott, A., S. V. Badman, S. W. H. Cowley, S. E. Milan, J. D. Nichols, and T. K. Yeoman (2009), Magnetosonic mach number dependence of the efficiency of reconnection between planetary and interplanetary magnetic fields, J. Geophys. Res., 114, A07219, doi:10.1029/2009JA014330.

Grocott, A., S. E. Milan, N. Sato, J. A. Wild, T. K. Yeoman, and A. S. Yukimatu (2010), Superposed epoch analysis of the ionospheric convection evolution during substorms: IMF $B_{Y}$ dependence, J. Geophys. Res., 115, A00I06, doi:10.1029/2010JA015728.

Heppner, J. P. (1972), The Harang discontinuity in auroral belt ionospheric currents, Geophys. Norv., 29, 105-120.

Heppner, J. P., and N. C. Maynard (1987), Empirical high-latitude electricfield models, J. Geophys. Res., 92(A5), 4467-4489.
Hughes, J., and W. Bristow (2003), SuperDarn observations of the Harang discontinuity during steady magnetospheric convection, J. Geophys. Res., 108(A5), 1185, doi:10.1029/2002JA009681.

Imber, S. M., S. E. Milan, and B. Hubert (2006), The auroral and ionospheric flow signatures of dual lobe reconnection, Ann. Geophys., 24, 3115-3129.

Imber, S. M., S. E. Milan, and B. Hubert (2007), Observations of significant flux closure by dual lobe reconnection, Ann. Geophys., 25(7), 1617-1627.

Kendall, M. G., A. Stuart, and J. K. Ord (1976), The Advanced Theory of Statistics, vol. 3, Design and Analysis, and Time-Series, Charles Griffin, London.

Khan, H., and S. W. H. Cowley (1999), Observations of the response time of high-latitude ionospheric convection to variations in the interplanetary magnetic field using EISCAT and IMP-8 data, Ann. Geophys., 17, 1306-1335, doi:10.1007/s005850050858.

Laundal, K. M., N. Ostgaard, K. Snekvik, and H. U. Frey (2010), Interhemispheric observations of emerging polar cap asymmetries, J. Geophys. Res., 115, A07230, doi:10.1029/2009JA015160.

Lukianova, R., and A. Kozlovsky (2011), IMF By effects in the plasma flow at the polar cap boundary, Ann. Geophys., 29, 1305-1315, doi:10.5194/angeo-29-1305-2011.

Milan, S. E., M. Lester, and S. W. H. Cowley (2000), Convection and auroral response to a southward turning of the IMF: Polar UVI, CUTLASS, and IMAGE signatures of transient magnetic flux transfer at the magnetopause, J. Geophys. Res., 105(A7), 15,741-15,755.

Pettigrew, E. D., S. G. Shepherd, and J. M. Ruohoniemi (2010), Climatological patterns of high-latitude convection in the Northern and Southern Hemispheres: Dipole tilt dependencies and interhemispheric comparisons, J. Geophys. Res., 115, A07305, doi:10.1029/2009JA014956.

Ruohoniemi, J. M., and K. B. Baker (1998), Large-scale imaging of highlatitude convection with Super Dual Auroral Radar Network HF radar observations, J. Geophys. Res., 103, 20,797-20,811, doi:10.1029/ 98JA01288.

Ruohoniemi, J. M., and R. A. Greenwald (1996), Statistical patterns of high-latitude convection obtained from Goose Bay HF radar observations, J. Geophys. Res., 101, 21,743-21,764, doi:10.1029/96JA01584.

Ruohoniemi, J. M., and R. A. Greenwald (2005), Dependencies of highlatitude plasma convection: Consideration of interplanetary magnetic field, seasonal, and universal time factors in statistical patterns, J. Geophys. Res., 110, A09204, doi:10.1029/2004JA010815.

Sandholt, P. E., and C. J. Farrugia (2007), Role of poleward moving auroral forms in the dawn-dusk auroral precipitation asymmetries induced by IMF By, J. Geophys. Res., 112, A04203, doi:10.1029/2006JA011952.

Shepherd, S., and J. Ruohoniemi (2000), Electrostatic potential patterns in the high-latitude ionosphere constrained by SuperDARN measurements, J. Geophys. Res., 105(A10), 23,005-23,014.

Shepherd, S. G., J. M. Ruohoniemi, and R. A. Greenwald (2003), Testing the Hill model of transpolar potential with Super Dual Auroral Radar Network observations, Geophys. Res. Lett., 30(1), 1002, doi:10.1029/ 2002GL015426.

Shi, J. K., et al. (2010), South-north asymmetry of field-aligned currents in the magnetotail observed by Cluster, J. Geophys. Res., 115, A07228, doi:10.1029/2009JA014446.

Smith, C. W., J. L'Heureux, N. F. Ness, M. H. Acuña, L. F. Burlaga, and J. Scheifele (1998), The ACE magnetic fields experiment, Space Sci. Rev., 86, 613-632, doi:10.1023/A:1005092216668. 
Stone, E. C., A. M. Frandsen, R. A. Mewaldt, E. R. Christian, D. Margolies, J. F. Ormes, and F. Snow (1998), The Advanced Composition Explorer, Space Sci. Rev., 86, 1-22, doi:10.1023/A:1005082526237.

Sun, W., W. Y. Xu, and S.-I. Akasofu (1998), Mathematical separation of directly driven and unloading components in the ionospheric equivalent currents during substorms, J. Geophys. Res., 103(A6), 11,695-11,700.
Xu, W. Y., and Y. Kamide (2004), Decomposition of daily geomagnetic variations by using method of natural orthogonal component, J. Geophys. Res., 109, A05218, doi:10.1029/2003JA010216. 